OPEN ACCESS

Edited by:

Brian Godman,

University of Strathclyde,

United Kingdom

Reviewed by:

Wania Cristina Da Silva,

Oswaldo Cruz Foundation (Fiocruz),

Brazil

Johanna Catharina Meyer, Sefako Makgatho Health Sciences

University, South Africa

${ }^{*}$ Correspondence:

Steven Simoens

steven.simoens@pharm.kuleuven.be

Specialty section:

This article was submitted to Pharmaceutical Medicine and

Outcomes Research,

a section of the journal

Frontiers in Pharmacology

Received: 11 November 2020

Accepted: 09 February 2021

Published: 12 April 2021

Citation:

Simoens S, Bento-Abreu A, Merckx B, Joubert S, Vermeersch $S$, Pavelyev A, Varga $S$ and Morais E (2021) Health Impact and Cost-Effectiveness of

Implementing Gender-Neutral Vaccination With the 9-Valent Human

Papillomavirus Vaccine in Belgium.

Front. Pharmacol. 12:628434.

doi: 10.3389/fphar.2021.628434

\section{Health Impact and Cost-Effectiveness of Implementing Gender-Neutral Vaccination With the 9-Valent Human Papillomavirus Vaccine in Belgium}

\author{
Steven Simoens ${ }^{1 *}$, Andre Bento-Abreu ${ }^{2}$, Barbara Merckx ${ }^{2}$, Sophie Joubert ${ }^{2}$, \\ Steve Vermeersch ${ }^{2}$, Andrew Pavelyev ${ }^{3,4}$, Stefan Varga ${ }^{3}$ and Edith Morais ${ }^{5}$ \\ ${ }^{1}$ KU Leuven Department of Pharmaceutical and Pharmacological Sciences, Leuven, Belgium, ${ }^{2}$ Merck Sharp \& Dohme, Brussels, \\ Belgium, ${ }^{3}$ Merck \& Co., Inc., Kenilworth, NJ, United States, ${ }^{4}$ HCL America, Inc., Sunnyvale, CA, United States, ${ }^{5}$ Merck Sharp \& \\ Dohme, Lyon, France
}

Background: Routine human papillomavirus (HPV) immunization in Belgium is currently regionally managed, with school-aged girls receiving the 9-valent HPV (9vHPV) vaccine in Flanders and Wallonia-Brussels with a national catch-up program for females only. This study will assess whether expanding these programs to gender-neutral vaccination (GNV) with the 9vHPV vaccine is a cost-effective strategy in Belgium.

Methods: A validated HPV-type transmission dynamic model estimated the potential health and economic impact of regional vaccination programs, comparing GNV versus female-only vaccination (FOV) with the 9vHPV vaccine in individuals aged 11-12 years in Flanders, GNV with the 9vHPV vaccine versus FOV with the 2-valent HPV (2vHPV) vaccine in individuals aged 12-13 years in Wallonia-Brussels, and national catch-up GNV versus FOV with the 9vHPV vaccine for those aged 12-18 years. Vaccination coverage rates of 90,50 , and $50 \%$ in both males and females were used in the base cases for the three programs, respectively, and sensitivity analyses were conducted. All costs are from the third-party payer perspective, and outcome measures were reported over a 100-year time horizon.

Results: GNV with the 9vHPV vaccine was projected to decrease the cumulative incidence of HPV 6/11/16/18/31/33/45/52/58-related diseases relative to FOV in both Flanders and Wallonia-Brussels. Further reductions were also projected for catch-up GNV with the 9vHPV vaccine, including reductions of $6.8 \%$ (2,256 cases) for cervical cancer, $7.1 \%$ (386 cases) and 18.8\% (2,784 cases) for head and neck cancer in females and males, respectively, and $30.3 \%$ (82,103 cases) and 44.6\% (102,936 cases) for genital warts in females and males, respectively. As a result, a GNV strategy would lead to reductions in HPV-related deaths. Both regional and national catch-up GNV strategies were projected to reduce cumulative HPV-related disease costs and were estimated to be cost-effective compared with FOV with incremental cost-effectiveness ratios of $€ 8,062$, $€ 4,179$, and $€ 6,127$ per quality-adjusted life-years in the three programs, respectively. Sensitivity analyses were consistent with the base cases. 
Conclusions: A GNV strategy with the 9vHPV vaccine can reduce the burden of HPVrelated disease and is cost-effective compared with FOV for both regional vaccination programs and the national catch-up program in Belgium.

Keywords: HPV, human papillomavirus, vaccine, cervical cancer, genital warts, cost-effectiveness, Belgium, 9-valent

\section{INTRODUCTION}

In 2007, the Superior Health Council of Belgium announced recommendations for human papillomavirus (HPV) vaccination for females aged 10 to 13 years and catch-up vaccination for females aged 14 to 26 years in addition to screening for the prevention of cervical cancer (Conseil Supérieur de la Santé, 2017; Thiry et al., 2019). HPV is a common sexually transmitted infection that can lead to the development of cervical, vulvar, and vaginal cancer in women, penile cancer in men, and genital warts, recurrent respiratory papillomatosis (RRP), and anal and head and neck cancers in both sexes (Schiffman and Castle, 2003; Forman et al., 2012; Giuliano et al., 2015; Fortes et al., 2017). HPV-related diseases impart a substantial burden on patients and their communities [The Belgian Health Care Knowledge Center (KCE), 2007]. In both Belgium and worldwide, there has been a trend for increasing HPV-related oropharyngeal cancer incidence, especially among males (incidences of 6.7 per 100,000 and 1.7 per 100,000 among males and females in Belgium, respectively (Bruni, 2017; de Martel et al., 2017). Furthermore, the estimated burden of cancers attributable to HPV 16/18 (excluding cervical cancer) in Europe has been shown to be higher in men than in women $(32.2 \%$ vs. $19.4 \%$, respectively, of new cases annually) and is driven primarily by head and neck cancers (26.4\% vs. $5.3 \%$, respectively) (Hartwig et al., 2012). Additionally, the likelihood of an HPV infection and rates of HPV-related disease (i.e., genital warts) and anal cancer are higher in male populations at high risk, such as men who have sex with men (MSM) (Nyitray et al., 2011; Anic et al., 2012; Frisch et al., 2003).

The importance of HPV vaccination is highlighted by the World Health Organization's global strategy to achieve 90-70-90 targets (90\% vaccine coverage, 70\% cervical screening rates, $90 \%$ cervical disease treatment rates) by 2030 in an attempt to eliminate cervical cancer (World Health Organization, 2020). As a result, many countries have implemented cervical cancer screening programs and HPV immunization programs to reduce the incidence of cervical cancer and other HPV-related diseases, including the manifestation of genital warts (Bruni et al., 2016; Bruni, 2017; Murillo et al., 2008). In 2017, the Belgian Superior Health Council updated its recommendations to propose HPV vaccination for immunocompromized patients, girls and boys aged 9 to 14, and catch-up vaccination for women and men aged 15 to 26 years old (Thiry et al., 2019)].

Vaccination in Belgium is made available through one of three options: reimbursement by the National Institute for Health and Disability Insurance, free of charge through regional community vaccination programs, or full price at pharmacies (Thiry et al., 2019). However, at the regional level, there are variations in the HPV vaccination recommendations and schedules (Figure 1).
Belgium is divided into three geographical regions-the Flanders region (population of 6.1 million people), the Wallonia region (3.4 million people), and the capital region of Brussels ( 1 million people) (Institut National d'Etudes demographiques, 2009). Currently, routine HPV immunization programs in Belgium are managed by the community governments in Federation Wallonia-Brussels and Flanders with national reimbursement available only for catch-up HPV vaccination for girls aged 12 to 18 who did not have access to vaccination through a regional vaccination program (Thiry et al., 2019). HPV immunization programs for females were initiated in both the Flanders and Wallonia-Brussels regions of Belgium in 2010 and 2011, respectively (Thiry et al., 2019).

Two HPV vaccines are currently available as part of the Belgium national reimbursement program: the 2-valent HPV (2vHPV; targeting HPV types 16/18) and the 9-valent HPV (9vHPV; targeting HPV 6/11/16/18/31/33/45/52/58) vaccines (Agentschap Zorg and Gezondheid, 2018). The 9vHPV vaccine provides the broadest coverage, targeting HPV types $6 / 11$, which are responsible for approximately $90 \%$ of genital warts cases globally (Garland et al., 2009); HPV types $16 / 18$, which are responsible for approximately $73 \%$ of cervical cancer, $13 \%$ of vulvar cancer, $50 \%$ of vaginal cancers, $80 \%$ of anal cancer, $23 \%$ of penile cancer, and $19 \%$ to $36 \%$ of oropharyngeal cancer in Europe; and HPV types 31/33/45/52/58, which are responsible for additional smaller percentages of HPVrelated cervical (16\%), vulvar (10\%), vaginal (15\%), anal (3\%), penile (12\%) and oropharyngeal cancers (4\%) in Europe (Nadiaye et al., 2014; Hartwig et al., 2017; de Sanjosé et al., 2018).

In Flanders, in 2010, the 4-valent HPV (4vHPV; targeting HPV $6 / 11 / 16 / 18)$ vaccine was made available for free in a 3-dose schedule through a regional vaccination program for females in the first year of secondary school (11-12 years old) (Geert, 2018). In 2014 , the program introduced the $2 \mathrm{vHPV}$ vaccine in a 2-dose schedule, then in July 2018, the 9vHPV vaccine (administered in a 2 -dose schedule) replaced the $2 \mathrm{vHPV}$ vaccine in the regional program. Vaccinating boys with the 9vHPV vaccine has been recommended since September 2017 and implemented since September 2019 (Vaccination Info Belgium, 2020a; Vaccination Info Belgium, 2020b). Vaccine uptake rates of approximately $90 \%$ in the Flanders region are for both two- and three-dose schedules of the HPV vaccine (Thiry et al., 2019).

In 2011, the 2vHPV vaccine was made available in the Wallonia-Brussels region through a free school-based program to females in the second year of secondary school (13-14-yearsold; initially in a 3-dose schedule and since 2014 in a 2-dose schedule) (Bruni, 2017; Thiry et al., 2019; Vaccination Info Belgium, 2020a). Vaccination of boys with the 9vHPV vaccine has been also implemented since September 2019. Vaccine uptake in the Wallonia-Brussels region is lower than for Flanders, 


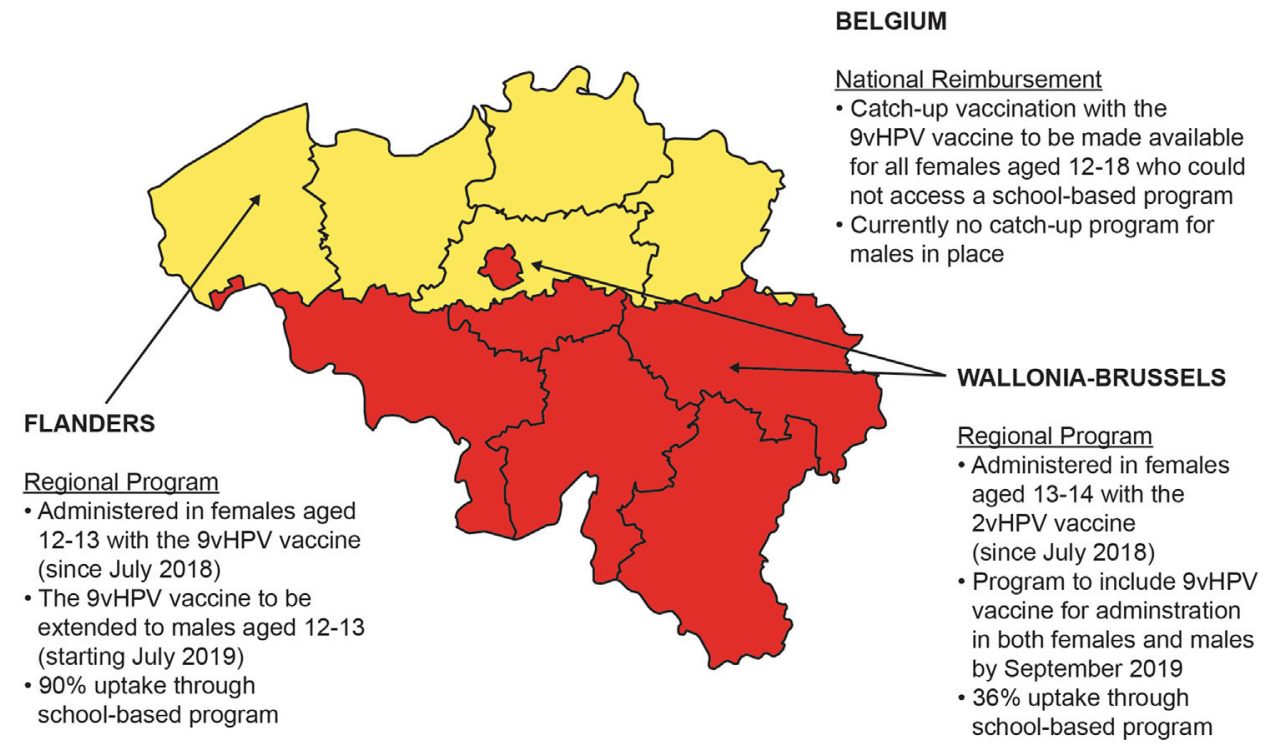

FIGURE 1 | National reimbursement and regional programs for HPV vaccination in Belgium.

estimated at $29.2 \%$ and $36.1 \%$ from 1 to 2 and 5 to 6 years after the start of the program, respectively (Thiry et al., 2019).

Both Flanders and the Wallonia-Brussels regions share similar age-standardized incidence rates for cervical cancer (7.3 and 8.8 cases per 100,000, respectively). However, the Flanders region has a higher mortality rate from cervical cancer $(1.9 / 100,000$ personyears compared to $1.2 / 100,000$ person-years in Wallonia) (Belgian Cancer Registry, 2020). In Belgium, the incidence of genital warts is high among men and women aged 23 to 30 years (145.82 and 220.11 per 100,000 person-years, respectively) (Dominiak-Felden et al., 2015). Furthermore, the incidence of head and neck cancer in Belgium is 3 times higher in males than in females (35.6 vs. 12.1 per 100,000 person-years) (Belgian Cancer Registry, 2017). However, limited data is available on the incidence of other HPV-related cancers in Belgium, with small retrospective studies reporting HPV was found in 22.3, 89.5 , and $61.1 \%$ of oropharyngeal squamous cell carcinoma, penile intraepithelial neoplasia, and invasive penile cancers, respectively (Grisar et al., 2016; D’Hauwers et al., 2012).

There is a strong rationale for immunizing females and males against HPV. Although a declining trend in the incidence/ prevalence of cervical cancer and other HPV-related diseases has been observed in females with the introduction of HPV vaccinations for girls in many countries, the burden of HPVrelated diseases in men remains high, particularly HPV-related anal and oral cancers (Dunne et al., 2007; Giuliano et al., 2008; Global Burden of Disease Cancer Collaboration et al., 2015). Additionally, men rarely acquire immunity following natural HPV infection, and antibodies acquired after an infection do not appear to protect against subsequent HPV infections or HPVrelated diseases (Beachler et al., 2018). Therefore, a GNV approach can directly protect males against HPV infection and HPV-related diseases and may further reduce HPV-related diseases in females. In recent years, several countries, most recently England, Germany, Netherlands, and France have introduced gender-neutral HPV vaccination to their national immunization programs to reduce the incidence of and burden associated with HPV-related diseases and cancers (Bruni, 2017; Takla et al., 2018; Kmietowicz, 2018).

Beginning in September 2019, both the Flanders and the Wallonia-Brussels regions in Belgium have switched to a gender-neutral HPV vaccination (GNV) program (Figure 1), joining in the international trend to combat HPV-related disease with HPV vaccination. In general, cost-effectiveness data are a key component in the decision-making process. A costeffectiveness analysis of GNV with the 2vHPV, 4vHPV, and 9vHPV vaccines in the United Kingdom setting demonstrated that a GNV approach was less cost-effective than a female-only vaccination (FOV) approach, most likely because of the United Kingdom's high vaccine uptake rates among females (2vHPV vaccine, $77-84 \%$; $4 \mathrm{vHPV}$ vaccine, $85-88 \%$ ) (Datta et al., 2019). A systematic review of cost-effectiveness studies evaluating GNV with the 9vHPV vaccine also indicated that the inclusion of adolescent boys into a vaccination program was costeffective only if vaccine price and coverage were low ( $\mathrm{Ng}$ et al., 2018). Recently, GNV with the 9vHPV vaccine was found to be cost-effective at high vaccine uptake rates $(80 \%)$ in European tender-based settings (Qendri et al., 2020). Furthermore, the same HPV disease transmission dynamic model was adapted to the French setting, demonstrating that GNV with the 9vHPV vaccine had a significant impact on public health benefits and may be considered more cost-effective than an FOV strategy (Majed et al., 2021). In the Belgium setting, it is not known whether expansion of existing FOV programs to a GNV strategy with the $9 \mathrm{vHPV}$ vaccine will be cost-effective. Therefore, the objective of this study is to assess the potential health and economic impact of a GNV strategy with the 9vHPV vaccine vs. FOV in all current $\mathrm{HPV}$ vaccination programs in Belgium. 


\section{MATERIAL AND METHODS}

\section{Mathematical Model \\ Design}

A previously validated and published HPV-type transmission dynamic model simulating the natural history of HPV infections and estimating the cost associated with HPV-related diseases in women has been extended to include all HPV-related diseases in both sexes (Elbasha et al., 2007; Elbasha \& Dasbach, 2010; Boiron et al., 2016; Largeron et al., 2017; Mennini et al., 2017; Kind et al., 2020; Majed et al., 2021). HPV infection and disease state transitions, lifetime durations of infection-derived immunity, and unvaccinated compartments in this model have been described previously in detail (Elbasha et al., 2007). was adapted to the Belgium setting in order to evaluate the health and economic impact of GNV with the 9vHPV vaccine versus FOV with the 9vHPV in the Flanders region and the $2 \mathrm{vHPV}$ in the Wallonia-Brussels region. The analysis also evaluated the impact of catch-up 9vHPV GNV compared to 9vHPV FOV on the population of Belgium (base-case analysis).

\section{Model Compartments}

The age-structured mathematical model (Elbasha et al., 2007) accounts for herd immunity and comprises three connected modules: 1) a demographic variables model describing birth, aging, and death; 2) a behavioral model describing sexual activity and sexual mixing patterns; and 3) a disease variables model describing screening rates and HPV infection and related disease transmission. In this model, individuals enter the population at a gender-specific and sexual activity-specific rate with an assumed age of sexual debut of 12 years and move between successive age groups at an age- and gender-specific rate per year (Elbasha et al., 2007). These individuals exit the model upon death at an age- and genderspecific per capita death rate per year (Elbasha et al., 2007). Patients with cervical cancer have an additional age- and stage-dependent death rate, but it was assumed that those with other HPV-related diseases [i.e., CIN, vaginal/vulvar cancers, vaginal intraepithelial neoplasia (VaIN), vulvar intraepithelial neoplasia (VIN), anal cancer, head and neck cancer, penile cancer, RRP, or genital warts] do not face an additional risk of death (Elbasha et al., 2007). The age-structured model also simulates HPV transmission and the occurrence of CIN, cervical cancer, external genital warts, and other HPV-related disease. The acquisition of an infection and progression of individuals from infection to disease follow a similar natural history structure, as assumed in previous models for HPV 16/18 (Dasbach et al., 2006). We also incorporated HPV 6/11/31/33/45/52/58 infections and associated diseases and grouped infections into HPV 16/18, HPV 6/11, or HPV 6/11/16/18/ $31 / 33 / 45 / 52 / 58$. The population was divided into distinct epidemiological categories according to the individual's status with respect to infection, disease, screening, and treatment (Elbasha et al., 2007). Further details of the model structure and assumptions have been published (Elbasha et al., 2007).

\section{Epidemiological Model Parameters}

Model input variables of the epidemiological model are divided into demographics, sexual behavior, disease and treatment patterns, and screening. A detailed summary of these parameter groups is provided in Supplementary Table S1 along with the data sources. When available, local Belgiumspecific data were used.

\section{Demographics}

Population figures and annual all-cause mortality rates for the general population by sex and age were retrieved from local Belgium-specific data (Supplementary Table S1). An overall Belgian population of $11,322,088$ individuals was included in the model, which comprised 5,754,083 females and 5,568,005 males (STATBEL, 2019). Annual all-cause mortality by age cohorts varied from $0.00075 \%$ among adolescent/adult males (15-44 years) to $0.09144 \%$ ( $\geq 75$ years) among the elderly male population, and from $0.00035 \%$ among adolescent/adult females (15-44 years) to $0.06844 \%$ ( $\geq 75$ years) among the elderly female population (La Belgique en Chiffres, 2019).

\section{Sexual Behavior}

Data from France were utilized for sexual behavior variables with the exceptions mentioned below (Supplementary Table S1). The annual mean number of sexual partners for age groups between 13 and $\geq 70$ years ranged from 0.0499 (13-14 years) to 1.50 (20-24 years) among males, and from 0.0778 (13-14 years) to 1.20 (18-29 years) among females (Bajos \& Boson et al., 2008); United States data were used for males aged 13-17 years and $\geq 70$ years (Elbasha and Dasbach, 2010). The proportions of the population at low (mean number of sexual partners per year of $0-1$ ), medium (2-4 sexual partners per year), and high (5 or more sexual partners per year) sexual activity risk were reported among males (87.71, 9.66, and $2.63 \%$, respectively) and females $(92.85,6.38$, and $0.77 \%$, respectively) (Bajos and Boson et al., 2008). The mean numbers of sexual partners per year by low, medium, and high sexual activity risk group were also reported among males $(0.86,2.65$, and 8.04 , respectively) and females $(0.84,2.58$, and 7.93 , respectively) (Bajos and Boson et al., 2008).

The degree of sexual mixing among members of different age cohorts and sexual activity groups ( 0 representing no mixing, and 1 representing a maximal mixing) were extracted from United States-specific data, (Elbasha and Dasbach, 2010) and was adjusted during the calibration process. Mixing between debut and cessation, and after cessation were 0.40 and 0.10 , respectively (Elbasha and Dasbach, 2010).

\section{Disease and Treatment Patterns}

Model input variables to simulate the natural history of the disease as well as disease and treatment patterns utilized local Belgium-specific data when available or estimated through model calibration (Supplementary Table S1).

Belgium-specific data on the incidence of HPV-related diseases were retrieved from previously published data and the Belgian Cancer Registry (Dominiak-Felden et al., 2015; Belgian Cancer Registry, 2020). Female incidence rates (per 100,000 person-years) for cervical cancer, vaginal cancer, vulvar cancer, anal cancer, head and neck cancer, and genital warts in Belgium were $11.12,0.73,3.80,2.27,10.78$, and 113.62 , respectively (Dominiak-Felden et al., 2015; Belgian Cancer Registry, 2020). 
TABLE 1 | Summary table on cancer mortality.

\begin{tabular}{|c|c|c|c|c|}
\hline \multirow[t]{2}{*}{ Cancer type } & \multirow[t]{2}{*}{ Age group (years) } & \multicolumn{3}{|c|}{ Annual mortality rate } \\
\hline & & Local cancer ${ }^{a}$ & Regional cancer ${ }^{a}$ & Distant cancer $^{a}$ \\
\hline \multirow[t]{5}{*}{ Cervical cancer } & $15-44$ & 0.009 & 0.030 & 0.075 \\
\hline & $45-54$ & 0.020 & 0.063 & 0.157 \\
\hline & $55-64$ & 0.029 & 0.094 & 0.236 \\
\hline & $65-74$ & 0.035 & 0.112 & 0.283 \\
\hline & $75+$ & 0.077 & 0.245 & 0.617 \\
\hline \multirow[t]{5}{*}{ Vaginal cancer } & $15-44$ & 0.033 & 0.057 & 0.102 \\
\hline & $45-54$ & 0.018 & 0.031 & 0.055 \\
\hline & $55-64$ & 0.048 & 0.083 & 0.148 \\
\hline & $65-74$ & 0.073 & 0.125 & 0.223 \\
\hline & $75+$ & 0.122 & 0.211 & 0.376 \\
\hline \multirow[t]{5}{*}{ Vulvar cancer } & $15-44$ & 0.026 & 0.057 & 0.124 \\
\hline & $45-54$ & 0.014 & 0.031 & 0.067 \\
\hline & $55-64$ & 0.038 & 0.083 & 0.179 \\
\hline & $65-74$ & 0.058 & 0.125 & 0.271 \\
\hline & $75+$ & 0.097 & 0.211 & 0.456 \\
\hline \multirow[t]{5}{*}{ Anal cancer (females) } & $15-44$ & 0.028 & 0.062 & 0.106 \\
\hline & $45-54$ & 0.033 & 0.074 & 0.127 \\
\hline & $55-64$ & 0.032 & 0.072 & 0.123 \\
\hline & $65-74$ & 0.043 & 0.095 & 0.163 \\
\hline & $75+$ & 0.084 & 0.187 & 0.321 \\
\hline \multirow[t]{5}{*}{ Anal cancer (males) } & $15-44$ & 0.031 & 0.068 & 0.118 \\
\hline & $45-54$ & 0.030 & 0.067 & 0.115 \\
\hline & $55-64$ & 0.040 & 0.090 & 0.154 \\
\hline & $65-74$ & 0.051 & 0.114 & 0.196 \\
\hline & $75+$ & 0.090 & 0.199 & 0.342 \\
\hline \multirow[t]{5}{*}{ Penile cancer } & $15-44$ & 0.008 & 0.037 & 0.082 \\
\hline & $45-54$ & 0.014 & 0.066 & 0.145 \\
\hline & $55-64$ & 0.013 & 0.061 & 0.134 \\
\hline & $65-74$ & 0.021 & 0.100 & 0.220 \\
\hline & $75+$ & 0.042 & 0.200 & 0.441 \\
\hline \multirow[t]{5}{*}{ Head and neck cancer (females) } & $15-44$ & 0.053 & 0.084 & 0.146 \\
\hline & $45-54$ & 0.069 & 0.109 & 0.191 \\
\hline & $55-64$ & 0.076 & 0.120 & 0.209 \\
\hline & $65-74$ & 0.088 & 0.139 & 0.242 \\
\hline & $75+$ & 0.155 & 0.244 & 0.427 \\
\hline \multirow[t]{5}{*}{ Head and neck cancer (males) } & $15-44$ & 0.066 & 0.132 & 0.235 \\
\hline & $45-54$ & 0.078 & 0.156 & 0.277 \\
\hline & $55-64$ & 0.088 & 0.175 & 0.311 \\
\hline & $65-74$ & 0.101 & 0.202 & 0.358 \\
\hline & $75+$ & 0.152 & 0.302 & 0.537 \\
\hline
\end{tabular}

aDisease stages can be related to the traditional TNM classification system as follows: "local disease" corresponds to stages I and II TNM classification (localized primary tumor); "regional disease" corresponds to stage III TNM classification system (metastasis to regional lymph nodes); "distant disease" corresponds to stage IV TNM classification system (distant metastatic disease), TNM, tumor-node-metastasis.

Male incidence rates for anal cancer, head and neck cancer, penile cancer, and genital warts in Belgium were 1.43, 31.10, 1.70, and 71.13, respectively (Belgian Cancer Registry, 2020; DominiakFelden et al., 2015). Incidence rates for the various HPV-related cancers and genital warts were estimated by applying the proportion of diseases attributable to all HPV types, and types in the 4vHPV and 9vHPV vaccines in Europe (Hartwig et al., 2017) with the exception of the proportion of HPV-related oropharynx cancers in Belgium, obtained from clinical experts (S. Nuyts, personal communication, February 21, 2019 and M. Remacle, personal communication, February 27, 2019).

Annual mortality rates for each HPV-related cancer, stratified by age and stage (local, regional, and distant disease), are summarized in Table 1. These were obtained by combining the data from two sources: survival data from the European
Cancer Registry (EUROCARE-5) database (2000 to 2007) and Cancer Survival data from the Belgian Cancer Registry (2004 to 2008) (Belgian Cancer Registry, 2020).

The proportions of the populations with HPV-related diseases (including cervical cancer [including the precancerous lesions cervical intraepithelial neoplasia $\{\mathrm{CIN} 1 / 2 / 3\}$ and carcinoma in situ $\{\mathrm{CIS}\}$ ], vaginal cancer [including the precancerous lesions VaIN $1 / 2 / 3$ ], vulvar cancer [including the precancerous lesions VIN $1 / 2 / 3$ ], anal cancer, genital warts, and head and neck cancer for females; and anal cancer, genital warts, penile cancer, head and neck cancer for males) recognizing their symptoms and seeking treatment, and the proportions who were treated were estimated through the model calibration. Similarly, the proportions of females and males who were treated for genital warts were estimated through the model calibration. 
TABLE 2 | Summary table on vaccine characteristics (Joura et al., 2007; Garland et al., 2007; Guiliano et al., 2011; Joura et al., 2015; Palefsky et al., 2011; Ault, 2007).

\begin{tabular}{ccccc}
\multicolumn{4}{c}{ Vaccine assumptions (percentage of exposed people avoiding the infection) } \\
\hline HPV type & HPV type & HPV type & HPV type & HPV types \\
6 & 11 & 16 & 18
\end{tabular}

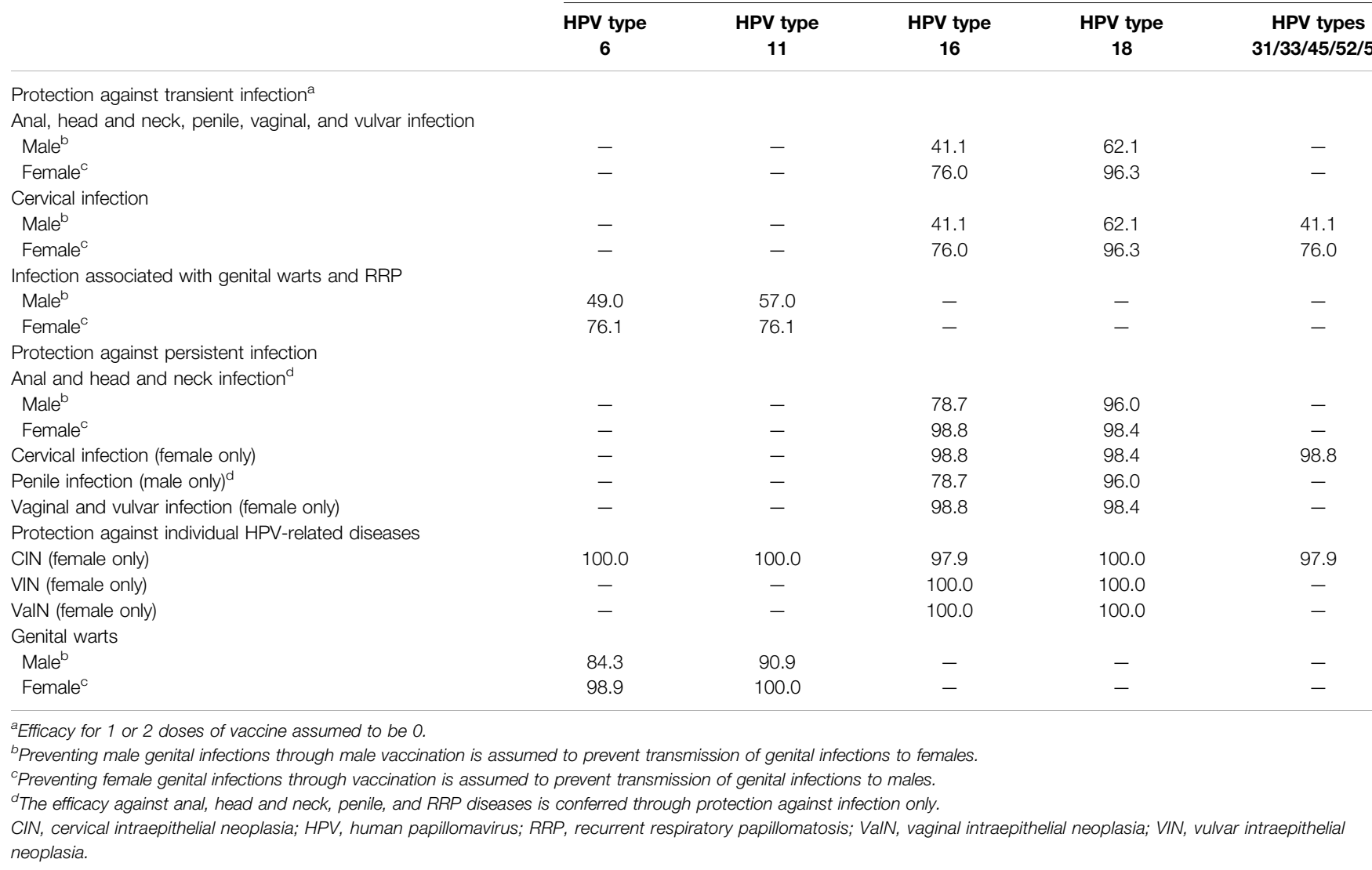

\section{Screening}

The proportion of females receiving a follow-up cervical cancer screening test after abnormal PAP smear diagnosis (90.21\%), and the proportion of females receiving gynecological cancer screening tests at least once every 3 years $(53.70 \%)$ were calculated based on data from the Belgian Healthcare Knowledge Center (KCE) and a 2007 expert Belgium survey (Supplementary Table S1). Cervical cancer screening adherence rates for females stratified by age were also calculated based on KCE data. In terms of diagnostic performance for cervical screening, the sensitivity and specificity of colposcopy were 96 and $48 \%$, respectively, whereas the specificity of the PAP screening test was $94 \%$ [The Belgian Health Care Knowledge Center (KCE), 2007]. In addition, the sensitivity of PAP screening for CIN was 63, 61, and $61 \%$ for CIN 1, CIN 2, and CIN 3, respectively (Arbyn et al., 2015).

\section{Vaccine Properties}

The prophylactic efficacy or the degree of protection offered by the vaccine was based on clinical trial data (Ault, 2007; Joura et al., 2007; Garland et al., 2007; Giuliano et al., 2011; Joura et al., 2015; Palefsky et al., 2011). The duration of protection against HPV genotypes 6/11/16/18/31/33/45/52/58 was assumed to be lifelong (except for the 20-year duration of protection in the sensitivity analysis) and herd immunity was assumed. Table 2 summarizes the vaccine efficacy parameters related to the protection against both transient and persistent infections.

\section{Economic Model Parameters}

The inputs for the economic model included vaccine strategy, vaccine properties, cost parameters, and health-related quality of life.

\section{Vaccine Strategies and Coverage Rates}

The vaccination schedule was assessed according to the product label, i.e., 2-dose schedule for children under 15 years and 3-dose schedule for children between 15 and 18 years old. Our base-case analyses were conducted according to three different scenarios: 1) in the Flanders region, strategy of GNV vs. FOV with 9vHPV vaccine administered as two doses in ages 11 to 12 years old; 2) in the Wallonia-Brussels region, strategy of GNV with the 9vHPV vaccine vs. FOV with the $2 \mathrm{vHPV}$ vaccine, both administered as two doses in ages 12 to 13 years old; and 3) in the national catchup program, strategy of GNV vs FOV with 9vHPV vaccine administered as two doses in ages 12 to 14 and 3 doses in ages 15 to 18 years old. 
TABLE 3 | Summary table on cost parameters for HPV-related diseases (third-party payer perspective; $2017 €$ ).

Parameter

Cost of vaccination

Per dose

9vHPV vaccine $\quad 123.97$

2VHPV vaccine $\quad 55.00$

Administration per dose

9vHPV vaccine

2vHPV vaccine

Total cost of single dose of vaccine plus administration

9vHPV vaccine

2vHPV vaccine

Cost per episode-of-care ${ }^{a}$

CIN 1 (female only)

CIN 2 (female only)

CIN 3 and CIS (female only)

Cervical cancer, local disease ${ }^{b}$ (female only)

Cervical cancer, regional disease ${ }^{\mathrm{b}}$ (female only)

Cervical cancer, distant disease ${ }^{\mathrm{b}}$ (female only)

ValN 2 (female only)

ValN 3 and CIS (female only)

Vaginal cancer, local disease ${ }^{\mathrm{b}}$ (female only)

Vaginal cancer, regional disease ${ }^{\mathrm{b}}$ (female only)

Vaginal cancer, distant disease ${ }^{b}$ (female only)

Vulvar cancer, local disease ${ }^{\mathrm{b}}$ (female only)

Vulvar cancer, regional disease ${ }^{b}$ (female only)

Vulvar cancer, distant disease ${ }^{\mathrm{b}}$ (female only)

Penile cancer, local disease ${ }^{b}$ (male only)

Penile cancer, regional disease ${ }^{b}$ (male only)

Penile cancer, distant disease ${ }^{\mathrm{b}}$ (male only)

Anal cancer, local disease ${ }^{b}$

Female

Male

Anal cancer, regional disease ${ }^{b}$

Female

Male

Anal cancer, distant disease ${ }^{\mathrm{b}}$

Female

Male

Head and neck cancer, local disease ${ }^{b}$

Female

Male

Head and neck cancer, regional disease ${ }^{b}$

Female

Male

Head and neck cancer, distant disease ${ }^{b}$

Female

Male

Genital warts

Female

Male

$\mathrm{RRP}^{\mathrm{c}}$

Female

Male

Screening and diagnostic tests ${ }^{d}$ (female only)

Screening (PAP smear and office visit)

Colposcopy

Biopsy

NA

NA

123.97

55.00

267.06

355.55

458.75

11,484

15,330

18,608

711

917

12,876

12,873

26,505

15,076

21,145

28,468

15,312

26,270

28,694

19,447

16,537

28,687

28,823

17,038

20,156

13,516

16,644

21,818

21,121

17,271

22,907

391

385

22,507

22,507

41

14

74
Cost $(2017 €)$

Data source

Belgium National institute for health and disability (INAMI) (2019)

Annemans et al. (2008)
Annemans et al. (2008)

Tsakeu et al. (2016)

Assumed twice as expensive as CIN costs

Tsakeu et al. (2016)

aInflation of 3.982\% (2015-2017) was applied (obtained from: statbel.fgov.be).

${ }^{b}$ Disease stages can be related to the traditional Tumor-Node-Metastasis (TNM) classification system as follows: "Iocal disease" corresponds to stages I and II TNM classification (localized primary tumor); "regional disease" corresponds to stage III TNM classification system (metastasis to regional lymph nodes); "distant disease" corresponds to stage IV TNM classification system (distant metastatic disease).

${ }^{c}$ Assumed that $3 \%$ was at the charge of the patient.

${ }^{d}$ For cervical and vaginal cancers only.

2vHPV, 2-valent human papillomavirus vaccine; 9vHPV, 9-valent human papillomavirus vaccine; CIN, cervical intraepithelial neoplasia; HPV, human papillomavirus; NA, not applicable; $R R P$, recurrent respiratory papillomatosis; ValN, vaginal intraepithelial neoplasia; VIN, vulvar intraepithelial neoplasia. 
GNV versus FOV with the 9vHPV vaccine in children ages 11 to 12 years was assessed at a $90 \%$ vaccine coverage rate (VCR) in Flanders, reflecting the high coverage in this region (Tjalma et al., 2018). GNV with the 9vHPV vaccine versus FOV with the $2 \mathrm{vHPV}$ vaccine in children aged 12 to 13 years was assessed at a VCR of $50 \%$ in the Wallonia-Brussels region. The national catch-up GNV versus catch-up FOV with the 9vHPV vaccine was assessed at a predicted VCR of $50 \%$ in males and females. This VCR is a 10 - to 15 -year projection allowing for the regional (school) program to provide protection for the entire population (catch-up cohort) analyzed in the base case and sensitivity scenarios. Catch-up vaccination was modeled in children aged 12 to 18 years to reflect the current national immunization program.

\section{Cost Parameters}

Cost parameters used in the model included cost per episode of care, cost of vaccination, and cost of screening and diagnostic tests; costs were reported in 2017 euros $(€)$. A detailed summary of all costs used in the model is provided in Table 3.

\section{Cost of Vaccination}

The base model adopted the public prices of vaccines in Belgium (€55 and $€ 123.97$ for the $2 \mathrm{vHPV}$ and 9vHPV vaccines, respectively).

\section{Cost Per Episode of Care}

The estimated costs per episode of care of each HPV-related disease, defined as the cost of management from diagnosis to resolution of the case, are summarized in Table 3. Costs were retrieved from Annemans et al. (Annemans et al., 2008) and realworld data from the IMS Health Belgian Hospital Disease Database (Hughes et al., 2011; Tsakeu et al., 2016).

\section{Cost of Screening and Diagnostic Tests}

For cervical and vaginal cancers, a 2016 report by the Belgian National Institute for Health and Disability Insurance (INAMI) was used to extract the cost associated with screening (PAP smear) and related office visits, whereas colposcopy and biopsy costs were retrieved from a 2015 update on HPV primary screening by the KCE (Arbyn et al., 2015). Costs for screening by PAP smear were set at $€ 41$, colposcopy at $€ 14$, and biopsy at $€ 74$.

\section{Perspective}

All costs are from the perspective of the third-party payer (i.e., only direct costs were included in the analysis).

\section{Discount Rates}

Discount rates of $1.5 \%$ for quality-adjusted life years (QALYs) and $3 \%$ for costs were applied to the model. These discount rates were in accordance with the Belgian KCE Guidelines for pharmaco-economic evaluation (Cleemput et al., 2008b).

\section{Health-Related Quality of Life}

The health utility values for the Belgian general population and for those with HPV-related diseases were derived from several sources. Health utilities for the Belgian general population by age and sex were based on data from the United Kingdom (Cleemput et al., 2004; Szende et al., 2014) and are summarized in Table 4. Disease-related health utilities for HPV-related diseases by sex and disease stage in the Belgian general population were estimated using United Kingdom- and United States-based data; these are also summarized in Table 4 (Szende and Williams et al., 2004; Hu \& Goldie, 2008; Chadha et al., 2010; Sullivan et al., 2011; Dominiak-Felden et al., 2013).

\section{Outcome Parameters Outcome Measures}

The model was used to estimate the following health outcome parameters: cumulative incidence of HPV-related diseases associated with HPV types targeted by the 9vHPV vaccine (6/ $11 / 16 / 18 / 31 / 33 / 45 / 52 / 58)$; number of prevented cases of HPVrelated diseases (expressed as the cumulative reduction in HPV 6/ $11 / 16 / 18 / 31 / 33 / 45 / 52 / 58$-related incident cases); number of prevented HPV-related deaths (expressed as the cumulative reduction in HPV 6/11/16/18/31/33/45/52/58-related incident cases). The model was also used to estimate the following economic outcome parameters: cumulative HPV-related disease health care costs; QALYs of the model population; and the incremental cost-effectiveness ratios (ICERs), which are calculated with the quotient: Incremental vaccination costs/ Incremental QALYs. Model calculations were performed using the mathematical software package Mathematica ${ }^{\circledR}$, Version 10.4 (Wolfram Research, Champaign, IL).

\section{Time Horizon}

Outcome measures were reported over a time horizon of 100 years because this was an appropriate time frame from which the system approached steady state and most benefits and costs of vaccination could be realized.

\section{Model Calibration and Validation}

Validation of the original model has been previously addressed (Elbasha et al., 2007; Elbasha and Dasbach, 2010). Consultation with experts on assumptions regarding the natural history of HPV infection and disease and vaccine characteristics was undertaken to establish face validity of the model. To confirm the predictive validity of the model, it was shown that model predictions of HPV prevalence and disease outcomes generally fell within the range of values reported in the literature. To assess the convergent validity of the original model, its estimates were compared with those of several previously published studies, revealing consistent predictions of population-level effectiveness and herd effects (Brisson et al., 2016). Results from the Elbasha model were consistent with those of other dynamic transmission models (Brisson et al., 2016).

The incidence rates for genital warts; CIN 1/2/3; and cervical, vaginal, vulvar, anal, penile, and head and neck cancers were calibrated.

All HPV-related diseases were considered, in accordance with the Belgian Superior Health Council recommendations and other health technology assessment agencies considering HPV vaccination (Takla et al., 2018; Conseil Supérieur de la Santé, 
TABLE 4 | Summary table on health utilities for individuals without HPV disease (by age) and for individuals with HPV-related diseases (by disease stage).

\begin{tabular}{|c|c|c|c|}
\hline & Females & Males & \\
\hline \multicolumn{4}{|l|}{ Individuals without HPV disease } \\
\hline \multicolumn{4}{|l|}{ Age group (years) } \\
\hline$<15$ & 0.849 & 0.850 & Szende and Williams (2004), Cleemput et al. (2004) \\
\hline $5-29$ & 0.849 & 0.850 & \\
\hline 30-39 & 0.816 & 0.837 & \\
\hline $40-49$ & 0.827 & 0.820 & \\
\hline $50-59$ & 0.801 & 0.798 & \\
\hline $60-69$ & 0.800 & 0.773 & \\
\hline $70-79$ & 0.733 & 0.714 & \\
\hline $80+$ & 0.686 & 0.730 & \\
\hline \multicolumn{4}{|l|}{ Individuals with HPV-related disease } \\
\hline $\mathrm{CIN} 1 / 2 / 3$ & 0.822 & NA & Hu and Goldie (2008), Sullivan et al. (2011) \\
\hline CIS (cervical) & 0.822 & NA & \\
\hline Cervical cancer, local & 0.822 & NA & \\
\hline Cervical cancer, regional & 0.732 & NA & \\
\hline Cervical cancer, distant & 0.542 & NA & \\
\hline Cervical cancer, cancer survivor & 0.822 & NA & \\
\hline ValN 2/3 & 0.822 & NA & \\
\hline CIS (Vaginal) & 0.822 & NA & \\
\hline Vaginal cancer, local & 0.822 & NA & \\
\hline Vaginal cancer, regional & 0.732 & NA & \\
\hline Vaginal cancer, distant & 0.542 & NA & \\
\hline Vaginal cancer, cancer survivor & 0.822 & NA & \\
\hline Vulvar cancer, local & 0.822 & NA & \\
\hline Vulvar cancer, regional & 0.732 & NA & \\
\hline Vulvar cancer, distant & 0.542 & NA & \\
\hline Vulvar cancer, cancer survivor & 0.822 & NA & \\
\hline Penile cancer, local & NA & 0.751 & \\
\hline Penile cancer, regional & NA & 0.661 & \\
\hline Penile cancer, distant & NA & 0.471 & \\
\hline Penile cancer, cancer survivor & NA & 0.751 & \\
\hline Anal cancer, local & 0.645 & 0.645 & \\
\hline Anal cancer, regional & 0.555 & 0.555 & \\
\hline Anal cancer, distant & 0.365 & 0.365 & \\
\hline Anal cancer, cancer survivor & 0.645 & 0.645 & \\
\hline Head and neck cancer, local & 0.756 & 0.756 & \\
\hline Head and neck cancer, regional & 0.666 & 0.666 & \\
\hline Head and neck cancer, distant & 0.476 & 0.476 & \\
\hline Head and neck cancer, cancer survivor & 0.756 & 0.756 & \\
\hline Genital warts & 0.900 & 0.900 & Dominiak-Felden et al. (2013) \\
\hline Recurrent respiratory papillomatosis & 0.760 & 0.760 & Chadha et al. (2010) \\
\hline
\end{tabular}

CIN, cervical intraepithelial neoplasia; CIS, carcinoma in situ; HPV, human papillomavirus; NA, not applicable; ValN, vaginal intraepithelial neoplasia.

2017; Favaretti et al., 2017; Joint committee on Vaccination and Immunization, 2018; Health Information and Quality Authority, 2018; KCE, 2019). Data were validated by national and international experts during an advisory board and conducted in agreement with the recent KCE report on HPV [The Belgian Health Care Knowledge Center (KCE), 2007].

\section{Sensitivity Analyses}

Deterministic one-way sensitivity analyses were conducted to assess the sensitivity of ICER values to variables that have been shown to be impactful to cost effectiveness. One variable is the VCR, which were assessed at different levels (5-95\% for male and female) (De La Fuente et al., 2019; Largeron et al., 2017). The lower VCR of 5\% in the national catch-up program is a closer representation of the current eligible population, when including all the school-aged males and some females who may not receive vaccination through the school program. Sensitivity analyses also assessed a genital warts incidence rate of 76 per 100,000 (low) and 250 per 100,000 (high); lower $(-20 \%)$ and higher $(+20 \%)$ incidence of HPV-related cancers; label-only indications for the 9vHPV vaccine (genital warts, precancerous lesions $[\mathrm{CIN} 1 / 2 / 3, \mathrm{VIN} 2 / 3, \mathrm{VaIN} 2 / 3$, anal intraepithelial neoplasia $1 / 2 / 3$ ], cervical, vulvar, vaginal, and anal cancers for females; and genital warts and anal cancer for males); duration of protection of 20 years; vaccine price discount of $\pm 10 \%$; utilities $( \pm 10 \%)$; GNV with 9vHPV vs. $2 \mathrm{vHPV}$; and FOV with 9vHPV versus FOV with 2vHPV.

\section{RESULTS}

\section{Regional Vaccination Program in Flanders}

Projected onto the population of Flanders, 9vHPV vaccination in both males and females demonstrated greater cumulative reductions in HPV 6/11/16/18/31/33/45/52/58-related diseases relative to FOV 
TABLE 5 | Cumulative reduction in HPV 6/11/16/18/31/33/45/52/58-related disease incidence for gender-neutral vaccination with the 9vHPV vaccine compared with 9vHPV vaccination in females only in the Flanders Region, 2vHPV Vaccination in Females Only in the Wallonia-Brussels regions, and 9vHPV Vaccination in Females Only nationwide in Belgium over 100 years.

\begin{tabular}{ccc}
\multicolumn{3}{c}{ Cumulative reduction in HPV 6/11/16/18/31/33/45/52/58-related incident } \\
cases over 100 years, n (\%) \\
\hline $\begin{array}{c}\text { Flanders region } \\
\text { (GNV 9vHPV vs. FOV }\end{array}$ & Wallonia-Brussels region & National catch-up \\
9vHPV) & (GNV 9vHPV vs. FOV & (GNV 9vHPV vs. FOV \\
2vHPV) & 9vHPV)
\end{tabular}

\begin{tabular}{|c|c|c|c|}
\hline \multicolumn{4}{|l|}{ Cervical } \\
\hline Cancer & $436(2.7)$ & $2,025(16.4)$ & $2,256(6.8)$ \\
\hline CIN 1 & $1,033(5.3)$ & $7,601(32.3)$ & $6,874(13.8)$ \\
\hline CIN 2/3 & $1,565(5.0)$ & $10,522(29.3)$ & $10,301(13.0)$ \\
\hline \multicolumn{4}{|l|}{ Vaginal } \\
\hline Cancer & $4(2.3)$ & $7(5.5)$ & $22(5.9)$ \\
\hline ValN 2/3 & $0(0.0)$ & $0(0.0)$ & $0(0.0)$ \\
\hline \multicolumn{4}{|l|}{ Vulvar } \\
\hline Cancer & $6(2.3)$ & $10(5.7)$ & $31(6.1)$ \\
\hline \multicolumn{4}{|c|}{ Genital warts and HPV 6/11-related CIN 1} \\
\hline Genital warts (female) & 30,288 (35.9) & 119,708 (65.2) & $82,103(30.3)$ \\
\hline Genital warts (male) & $53,538(60.1)$ & $73,421(63.1)$ & $102,936(44.6)$ \\
\hline $\mathrm{CIN} 1$ & $3,564(35.5)$ & $12,117(61.6)$ & $9,018(28.6)$ \\
\hline \multicolumn{4}{|l|}{ Anal } \\
\hline Cancer (female) & $132(3.7)$ & $182(7.7)$ & $553(7.4)$ \\
\hline Cancer (male) & 429 (16.5) & $294(17.7)$ & $953(17.8)$ \\
\hline \multicolumn{4}{|l|}{ Head and neck } \\
\hline Cancer (female) & 133 (3.6) & $155(6.4)$ & $386(7.1)$ \\
\hline Cancer (male) & 2,346 (19.1) & 1,391 (18.0) & 2,784 (18.8) \\
\hline \multicolumn{4}{|l|}{ Penile } \\
\hline Cancer & 490 (28.6) & 206 (20.8) & $461(22.1)$ \\
\hline \multicolumn{4}{|l|}{ RRP } \\
\hline Female & 97 (38.4) & $343(65.1)$ & $244(31.0)$ \\
\hline Male & $137(52.1)$ & $270(63.7)$ & $284(38.4)$ \\
\hline
\end{tabular}

2vHPV, 2-valent human papillomavirus vaccine; 9vHPV, 9-valent human papillomavirus vaccine; CIN, cervical intraepithelial neoplasia; FOV, female-only vaccination; GNV, gender-neutral vaccination; HPV, human papillomavirus; RRP, recurrent respiratory papillomatosis; ValN, vaginal intraepithelial neoplasia.

TABLE 6 | Cumulative reduction in HPV 6/11/16/18/31/33/45/52/58-related deaths over 100 years with 9vHPV vaccination in females and males compared with femaleonly vaccination.

Cumulative reduction in HPV 6/11/16/18/31/33/45/52/58-related deaths, $n$ (\%)

\begin{tabular}{cc}
\hline Flanders (GNV 9vHPV & Wallonia-Brussels (GNV 9vHPV \\
vs. FOV 9vHPV) & vs. FOV 2vHPV)
\end{tabular}

\begin{tabular}{|c|c|c|c|}
\hline & $\begin{array}{c}\text { Flanders (GNV 9vHPV } \\
\text { vs. FOV 9vHPV) }\end{array}$ & $\begin{array}{c}\text { Wallonia-Brussels (GNV 9vHPV } \\
\text { vs. FOV 2vHPV) }\end{array}$ & $\begin{array}{c}\text { National catch-up (GNV } \\
\text { 9vHPV vs. FOV } \\
\text { 9vHPV) }\end{array}$ \\
\hline Cervical cancer & $127(2.5)$ & $525(14.1)$ & $594(5.8)$ \\
\hline Vaginal cancer & $1(2.1)$ & $1(4.7)$ & $5(5.0)$ \\
\hline Vulvar cancer & $2(2.1)$ & $2(5.0)$ & $8(5.2)$ \\
\hline Anal cancer (female) & $40(3.3)$ & $53(6.8)$ & $160(6.5)$ \\
\hline Anal cancer (male) & $81(14.8)$ & $56(15.9)$ & $176(15.9)$ \\
\hline Head and neck cancer (female) & $43(3.3)$ & $48(5.7)$ & $120(6.4)$ \\
\hline Head and neck cancer (male) & $1,175(18.0)$ & $688(17.0)$ & 1,379 (17.8) \\
\hline Penile cancer & $67(25.2)$ & $29(18.5)$ & $63(19.7)$ \\
\hline RRP (female) & $4(31.5)$ & $15(61.5)$ & $11(27.7)$ \\
\hline RRP (male) & $6(44.5)$ & $12(60.2)$ & $13(34.8)$ \\
\hline
\end{tabular}

2vHPV, 2-valent human papillomavirus vaccine; 9vHPV, 9-valent human papillomavirus vaccine; FOV, female-only vaccination; GNV, gender-neutral vaccination; HPV, human papillomavirus; RRP, recurrent respiratory papillomatosis.

over a 100-year period (Table 5). GNV with the 9vHPV vaccine is projected to decrease the cumulative incidence of anal, penile, and head and neck cancers in males by 429 (16.5\%), 490 (28.6\%), and $2,346(19.1 \%)$ cases, respectively. Notably, GNV is also projected to benefit females, by reducing incidence of cervical cancer by 436 cases (2.7\%), CIN 1 by 1,033 cases (5.3\%), CIN $2 / 3$ by 1,565 cases $(5.0 \%)$, and HPV 6/11-related CIN 1 by 3,564 cases (35.5\%). In addition, a cumulative incidence of genital warts was projected to decrease by $53,538(60.1 \%)$ and $30,288(35.9 \%)$ cases in males and females, respectively.

As a result of reductions in the incidence of HPV-related diseases, the model predicts cumulative reduction in HPV 6/11/ 
TABLE 7 | Estimated reductions in cumulative HPV-related disease costs over 100 years with 9vHPV vaccination in females and males compared with female-only vaccination.

\begin{tabular}{|c|c|c|c|c|c|c|c|c|c|}
\hline & \multicolumn{9}{|c|}{ Cumulative HPV 6/11/16/18/31/33/45/52/58-related disease costs over 100 years (2017 $€$ ) } \\
\hline & \multicolumn{3}{|c|}{ Flanders } & \multicolumn{3}{|c|}{ Wallonia-Brussels } & \multicolumn{3}{|c|}{ National catch-up } \\
\hline & FOV 9vHPV & GNV 9vHPV & $\begin{array}{c}\text { \% Reduction } \\
\text { with GNV }\end{array}$ & FOV 2vHPV & GNV 9vHPV & $\begin{array}{c}\text { \% Reduction } \\
\text { with GNV }\end{array}$ & FOV 9vHPV & GNV 9vHPV & $\begin{array}{c}\text { \% Reduction } \\
\text { with GNV }\end{array}$ \\
\hline \multicolumn{10}{|l|}{ Cervical } \\
\hline Cancer & $121,463,664$ & $120,090,179$ & 1.1 & $75,110,163$ & $70,838,451$ & 5.7 & $221,068,799$ & $215,727,358$ & 2.4 \\
\hline $\mathrm{CIN} 1$ & 646,876 & 627,818 & 2.9 & 511,252 & 425,167 & 16.8 & $1,245,220$ & $1,166,577$ & 6.3 \\
\hline CIN 2/3 & $5,582,165$ & $5,432,369$ & 2.7 & $4,208,443$ & $3,673,786$ & 12.7 & $10,762,979$ & $10,149,886$ & 5.7 \\
\hline \multicolumn{10}{|l|}{ Vaginal } \\
\hline Cancer & $1,635,803$ & $1,621,481$ & 0.9 & 935,144 & 918,495 & 1.8 & $2,923,035$ & $2,866,547$ & 1.9 \\
\hline ValN 2/3 & 0 & 0 & 0 & 0 & 0 & 0 & 0 & 0 & 0 \\
\hline \multicolumn{10}{|l|}{ Vulvar } \\
\hline Cancer & $2,669,528$ & $2,645,119$ & 0.9 & $1,534,649$ & $1,506,204$ & 1.9 & $4,780,932$ & $4,684,450$ & 2.0 \\
\hline \multicolumn{10}{|c|}{ Genital warts and HPV 6/11-related CIN 1} \\
\hline $\mathrm{CIN} 1$ & 244,805 & 205,711 & 16.0 & 261,698 & 150,875 & 42.3 & 529,205 & 438,059 & 17.2 \\
\hline Genital warts (male) & $15,056,393$ & $10,019,758$ & 33.5 & $14,357,243$ & $7,638,673$ & 46.8 & $31,993,851$ & $22,026,928$ & 31.2 \\
\hline Genital warts (female) & $18,983,387$ & $15,698,664$ & 17.3 & $23,020,233$ & $11,888,494$ & 48.4 & $42,129,959$ & $33,846,351$ & 19.7 \\
\hline \multicolumn{10}{|l|}{ Anal } \\
\hline Cancer (male) & $21,298,329$ & $20,035,436$ & 5.9 & $12,437,627$ & $11,636,169$ & 6.4 & $40,377,601$ & $37,660,056$ & 6.7 \\
\hline Cancer (female) & $30,871,133$ & $30,498,619$ & 1.2 & $18,513,400$ & $18,064,295$ & 2.4 & $58,346,553$ & $56,959,678$ & 2.4 \\
\hline \multicolumn{10}{|l|}{ Head and neck } \\
\hline Cancer (male) & $106,565,385$ & $99,134,494$ & 7.0 & $60,876,996$ & $56,765,509$ & 6.8 & $118,112,189$ & $109,546,861$ & 7.3 \\
\hline Cancer (female) & $27,060,966$ & $26,732,398$ & 1.2 & $15,842,344$ & $15,519,383$ & 2.0 & $35,862,224$ & $35,033,232$ & 2.3 \\
\hline \multicolumn{10}{|l|}{ Penile } \\
\hline Cancer & $11,495,546$ & $10,322,974$ & 10.2 & $6,597,901$ & $6,083,218$ & 7.8 & $13,692,130$ & $12,518,954$ & 8.6 \\
\hline RRP & $6,034,979$ & $4,701,432$ & 22.1 & $6,861,054$ & $3,579,656$ & 47.8 & $13,224,158$ & $10,215,656$ & 22.8 \\
\hline Total disease costs & $369,608,960$ & $347,757,452$ & 5.9 & $241,068,147$ & $208,688,374$ & 13.4 & $595,048,835$ & $552,840,593$ & 7.1 \\
\hline
\end{tabular}

2vHPV, 2-valent human papillomavirus vaccine; 9vHPV, 9-valent human papillomavirus vaccine; CIN, cervical intraepithelial neoplasia; FOV, female-only vaccination; GNV, gender-neutral vaccination; HPV, human papillomavirus; RRP, recurrent respiratory papillomatosis.

16/18/31/33/45/52/58-related deaths over 100 years (Table 6), with the greatest reduction in deaths from head and neck cancer ( 1,175 for males and 43 for females, respectively), cervical cancer (127), anal cancer (81 for males and 40 for females), and penile cancer (67).

Upon evaluating the economic impact of the associated projected reductions in incidence associated with GNV relative to FOV with the 9vHPV vaccine, the Flanders region is expected to see a $5.9 \%$ reduction $(€ 21,851,508)$ in cumulative HPV 6/11/ 16/18/31/33/45/52/58-related disease costs (Table 7); 54.1 and $44.4 \%$ of the reduction in cumulative HPV 6/11/16/18/31/33/45/ 52/58-related disease costs were attributable to HPV 16/18- and HPV 6/11-related disease costs, respectively.

GNV with the 9v HPV vaccine is a cost-effective strategy versus FOV with the 9vHPV vaccine in the Flanders region, with an ICER of $€ 8,062$ per QALY. Sensitivity analyses projected that decreasing the VCR improves ICER, with a 50\% VCR leading to an ICER of $€ 4,042$ per QALY, whereas boosting the VCR to $95 \%$ increases the ICER to $€ 8,854$ per QALY (Figure 2A). Decreasing the duration of protection of the $9 \mathrm{vHPV}$ vaccine (assumed to be lifelong in the base case) to 20 years would have minimal impact on the ICER ( $€ 8,024$ per QALY). Assuming a high genital wart incidence will decrease the ICER to $€ 6,974$ per QALY, whereas assuming a low incidence will increase the ICER to $€ 8,376$ per QALY. Limiting the model to only diseases included in the 9vHPV vaccine label leads to an ICER of $€ 40,847$ per QALY (Figure 2A).

\section{Regional Vaccination Program in Wallonia-Brussels}

In the Wallonia-Brussels region, GNV with the 9vHPV was also estimated to provide greater cumulative reductions in HPV6/11/16/18/31/33/45/52/58-related disease compared with FOV with the 2vHPV vaccine (Table 5). The model projected that GNV with the 9vHPV vaccine would decrease the cumulative incidence of anal, penile, and head and neck cancers in males by 294 (17.7\%), 206 (20.8\%), and 1,391 (18.0\%) cases, respectively. In females, an additional 2,025 cases of cervical cancer (16.4\%), 7,601 cases of CIN1 (32.3\%), 10,522 cases of CIN 2/3 (29.3\%), and 12,117 cases of HPV 6/11-related CIN1 (61.6\%) would be prevented relative to FOV with the $2 \mathrm{vHPV}$ vaccine. Cumulative incidence of genital warts was projected to decrease by $73,421(63.1 \%)$ and 119,708 (65.2\%) cases in males and females, respectively.

The model also projected the greatest number of averted deaths would be for head and neck cancer (688 for males and 48 for females), cervical cancer (525), anal cancer (56 for males and 53 for females), and penile cancer (29) (Table 6).

In the Wallonia-Brussels region, GNV with the 9vHPV vaccine was estimated to result in a $13.4 \%(€ 32,379,773)$ 
A

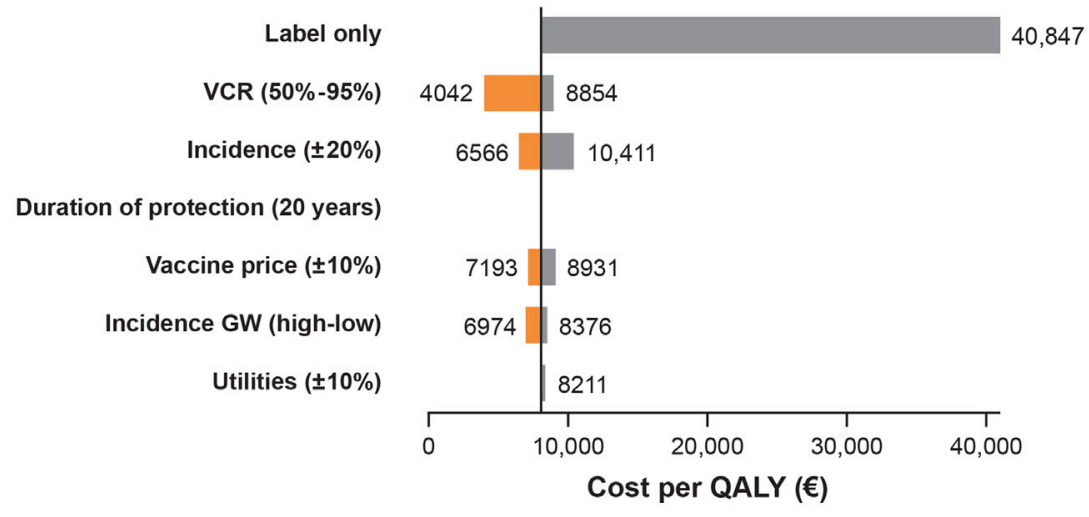

B $\quad$ GNV 9vHPV vs FOV 2vHPV in Wallonia-Brussels

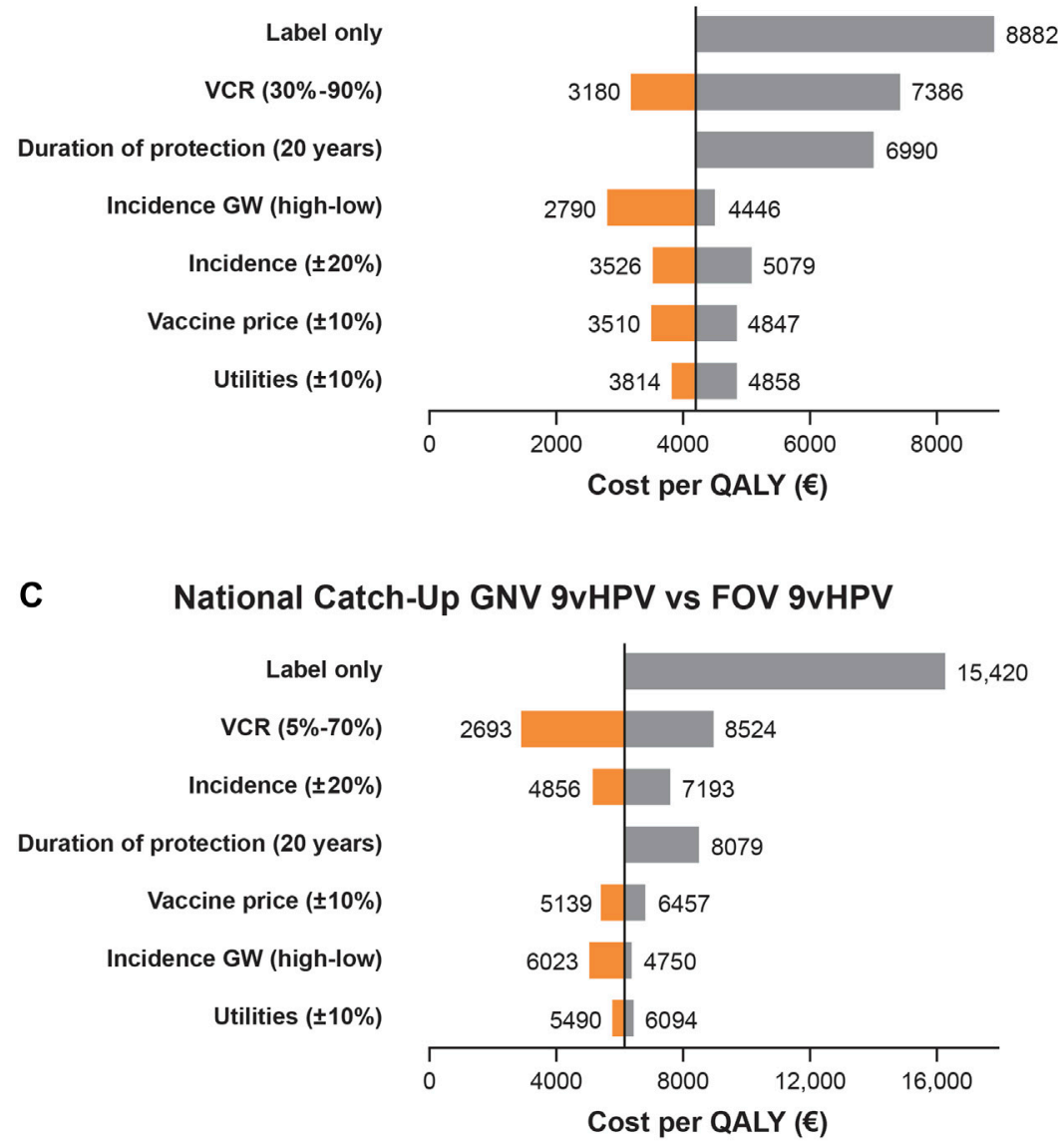

FIGURE 2 | Univariate sensitivity analyses of the base case for the incremental cost-effectiveness ratio (ICER; cost per QALY) of gender-neutral vaccination (GNV) with the 9VHPV vaccine in the Flanders Region of Belgium versus female-only vaccination [FOV] with the 9vHPV Vaccine) (A); in the Wallonia-Brussels region of Belgium versus FOV with the 2vHPV vaccine (B); and as part of a national HPV vaccine catch-up program compared with FOV with the 9vHPV vaccine in Belgium (C) over

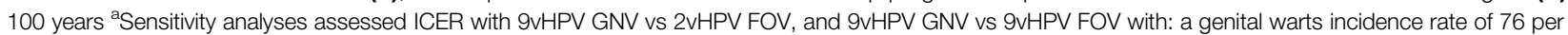
100,000 (low) and 250 per 100,000 (high); lower (-20\%) and higher (+20\%) incidence of HPV-related cancers; label-only indications for the 9vHPV vaccine (genital warts, precancerous lesions [CIN 1/2/3, VIN 2/3, ValN 2/3, anal intraepithelial neoplasia 1/2/3], cervical, vulvar, vaginal, and anal cancers for females; and genital warts and anal cancer for males); duration of protection of 20 years; vaccine price discount of $\pm 10 \%$; and utilities of $\pm 10 \%$. 
reduction in HPV6/11/16/18/31/33/45/52/58-related disease costs after 100 years compared with FOV with the $2 \mathrm{vHPV}$ vaccine (Table 7); 23.4 and $65.6 \%$ of the reductions in cumulative HPV 6/11/16/18/31/33/45/52/58-related disease costs were attributable to HPV 16/18- and HPV 6/11-related disease costs, respectively.

GNV with the 9v HPV vaccine is a cost-effective strategy versus FOV with the $2 \mathrm{vHPV}$ vaccine, with an ICER of $€ 4,179$ per QALY, well below previously estimated ICERs for FOV conducted in $2007(€ 33,000)$ (Thiry et al., 2019). Sensitivity analyses projected that decreasing the VCR improves ICER, with a $30 \%$ VCR leading to an ICER of $€ 3,180$ per QALY, whereas boosting the VCR to $90 \%$ increases the ICER to $€ 7,386$ per QALY (Figure 2B). Decreasing the duration of protection of the 9vHPV vaccine (assumed to be lifelong in the base case) to 20 years increases the ICER to $€ 6,990$ per QALY. Assuming a high genital wart incidence will decrease the ICER to $€ 2,790$ per QALY, whereas assuming a low incidence will increase the ICER to $€ 4,446$ per QALY. Limiting the model to only diseases included in the 9vHPV vaccine label leads to an ICER of $€ 8,882$ per QALY, well below the thresholds commonly used for cost-effectiveness evaluations (Figure 2B) (National Institute for Health and Care Excellence, 2014; Bertram et al., 2016; Thiry et al., 2019).

\section{National Catch-Up HPV Vaccination Reimbursement Program}

Catch-up HPV vaccination with the 9vHPV vaccine through the Belgian national program in both males and females demonstrated greater cumulative reductions in HPV 6/11/16/ $18 / 31 / 33 / 45 / 52 / 58$-related diseases relative to FOV with the $9 \mathrm{vHPV}$ vaccine over a 100 -year period (Table 5), reducing the projected incidence of cervical cancer and penile cancer by 6.8 and $22.1 \%$. Furthermore, additional decreases in HPV-related disease incidence for head and neck cancer and anal cancer were projected in both males (18.8 and $17.8 \%$, respectively) and females (7.1 and 7.4\%, respectively). A GNV catch-up strategy with the 9vHPV vaccine was also estimated to reduce genital warts incidence by 44.6 and $30.3 \%$ in males and females, respectively, relative to FOV.

The model estimated that the greatest number of averted HPV-related cancer deaths were for head and neck cancer $(1,379$ [17.8\%] for males and 120 [6.4\%] for females) and cervical cancer $(594[5.8 \%])$ after 100 years of catch-up GNV relative to FOV with the 9vHPV vaccine (Table 6).

In accordance with the projected reductions in HPV-related disease burdens associated with the catch-up GNV relative to FOV with the 9vHPV vaccine, the model projected a $7.1 \%$ reduction $(€ 42,208,242)$ in HPV 6/11/16/18/31/33/45/52/58related disease costs over a 100-year period (Table 7). Including only HPV-related diseases that are in HPV vaccine labels in the model resulted in a similar projected $6.9 \%$ decrease $(€ 28,632,227)$ in HPV 6/11/16/18/31/33/45/52/58-related disease costs; 46.4 and $50.6 \%$ of the reductions in cumulative HPV 6/11/ $16 / 18 / 31 / 33 / 45 / 52 / 58$-related disease costs were attributable to HPV 16/18- and HPV 6/11-related disease costs.
Catch-up GNV with the 9v HPV vaccine is a cost-effective strategy versus FOV with the 9vHPV vaccine, with an ICER of $€ 6,127$ per QALY. Sensitivity analyses projected that decreasing the VCR improves ICER, with a 5\% VCR leading to an ICER of $€ 2,866$ per QALY, whereas boosting the VCR to $70 \%$ increases the ICER to $€ 8,990$ per QALY (Figure 2C). Decreasing the duration of protection of the 9vHPV vaccine (assumed to be lifelong in the base case) to 20 years increases the ICER to $€ 8,522$ per QALY. Assuming a high genital wart incidence will decrease the ICER to $€ 5,043$ per QALY, whereas assuming a low incidence will increase the ICER to $€ 6,361$ per QALY. Limiting the model to only diseases included in the 9vHPV vaccine label leads to an ICER of $€ 16,259$ per QALY, still below the thresholds commonly used for cost-effectiveness evaluations (Figure 2C) (National Institute for Health and Care Excellence, 2014; Bertram et al., 2016; Thiry et al., 2019).

\section{DISCUSSION}

This analysis included evaluation of the health impact (i.e., HPVrelated disease incidence and death) and cost-effectiveness of HPV GNV with 9vHPV vaccine on HPV-related diseases based on recommendations from the Superior Health Council of Belgium as well as other health technology assessment agencies (KCE, 2019; Conseil Supérieur de la Santé, 2017; Health Information and Quality Authority, 2018). Using a validated transmission dynamic model, GNV with the 9vHPV vaccine, whether as part of the regional immunization programs in Flanders and Wallonia-Brussels or the Belgian national catchup HPV vaccination program, is projected to reduce HPV-related disease incidence and health care costs relative to FOV with either the $9 \mathrm{vHPV}$ or the $2 \mathrm{vHPV}$ vaccine. Based on the model outcomes, vaccinating boys is projected to reduce anal cancer and genital wart incidence in males, and also provide additional protection for females with substantial reductions in the incidence of cervical cancer, precancerous cervical lesions, anal cancer, and genital warts. Although HPV vaccines are not indicated for the prevention of RRP, head and neck cancer, and penile cancer as per label (Gardasil, 2018), there is evidence demonstrating the efficacy of HPV vaccines in reducing persistent HPV infections at these anatomic sites (e.g., persistent external genital infection (Giuliano et al., 2011), persistent oral infection (Herrero et al., 2013; Wilkin et al., 2018), and many countries and international literature considered that HPV vaccines may protect against these diseases, (Takla et al., 2018; Burger et al., 2014; Chesson et al., 2016; Joint committee on Vaccination and Immunization, 2018; Mauz et al., 2018; Boiron et al., 2016; Matsuzaki et al., 2020). Our model suggests GNV with the 9vHPV vaccine is a cost-effective strategy in Flanders (vs. FOV with the 9vHPV vaccine), Wallonia-Brussels (vs. FOV with the $2 \mathrm{vHPV}$ vaccine), and the Belgium national catchup program (vs. FOV with the 9vHPV vaccine), with the ICERs being $€ 8,062$, $€ 4,179$, and $€ 6,127$ in the three programs, respectively. The difference in the ICERs of the Flanders and Wallonia-Brussels regions is the result of the different VCRs assumed in our model based on the VCRs of the current vaccination programs in these regions (approximately 
90 and $36 \%$ in the Flanders and Wallonia-Brussels regions, respectively) (Tjalma et al., 2018). The sensitivity analyses demonstrated that switching to GNV with the 9vHPV vaccine is cost-effective regardless of the VCR situation analyzed (30-95\% for the regional programs and $5-70 \%$ for the national catch-up program).

Although there is no official cost-effectiveness threshold in Belgium, (Cleemput et al., 2008a; Cleemput et al., 2012) a threshold value of $€ 33,000$ per QALY gained was used in a recent KCE cost-effectiveness analysis of HPV vaccination in Belgium (Thiry et al., 2019). This value falls between the World Health Organization threshold range of 1 to 3 times the per capita gross domestic product (GDP) of the country per additional Disability Adjusted Life Years (Belgium, $€ 37,532$ to $€ 112,597)$ and the United Kingdom National Institute for Health and Care Excellence threshold range of cost-effectiveness ( $£ 20,000$ to $£ 30,000$, or $€ 23,000$ to $€ 34,000$ per additional QALY) (National Institute for Health and Care Excellence, 2014; Bertram et al., 2016; Thiry et al., 2019). ICERs predicted by our model for Flanders, Wallonia-Brussels, and the Belgian national GNV catchup program were well below these thresholds, thus highlighting that GNV with the 9vHPV vaccine is a very cost-effective strategy in Belgium.

Our model includes all HPV-related diseases (i.e., cervical cancer [including CIN $1 / 2 / 3$ and carcinoma in situ], vaginal cancer [including VaIN 1/2/3], vulvar cancer [including VIN $1 / 2 / 3$ ], anal cancer, head and neck cancer, and penile cancer), although sensitivity analyses show that GNV with the 9vHPV is still cost-effective relative to FOV with either the 2vHPV or the $9 \mathrm{vHPV}$ vaccines when only considering indications in the label. As per label, HPV vaccines are not indicated for the prevention of RRP, head and neck cancer, and penile cancer (Gardasil, 2018).

The reductions in RRP projected in these analyses are of interest. RRP is a rare, chronic disease associated with severe morbidity and in some cases mortality (Fortes et al., 2017). HPV types 6 and 11 are responsible for more than $90 \%$ of cases of RRP (Donne et al., 2010; Fortes et al., 2017). The age distribution for RRP is bimodal, with a juvenile form likely due to exposure to HPV in the peripartum period and an adult form caused by HPV infection in adulthood (Fortes et al., 2017). A systematic review looking at RRP incidence rates in Norway reported an adult rate of 0.23 per 100,000 and 0.88 per 100,000 in females and males, respectively, and a juvenile incidence of 0.09 per 100,000 in females and 0.26 per 100,000 in males (Omland et al., 2012). Furthermore, RRP has no ICD code, which makes it difficult to identify cases and to measure its incidence. Real-world data indicate a benefit of HPV vaccination for preventing and treating RRP (Yiu et al., 2019; Novakovic et al., 2018). In Australia, an HPV vaccination catch-up program with the $4 \mathrm{vHPV}$ vaccine has been available since 2007 to all schoolgirls aged 12-13 years, with a catch-up program for older schoolgirls and women up to 26 years of age; schoolboys were subsequently added to the vaccination program in 2013 (Donovan and Callander, 2018). As a result, a clear reduction in the incidence of RRP of at least 8 times between 2012 and 2016 was demonstrated in a prospective study evaluating the impact of this national
HPV vaccination program (Novakovic et al., 2018). Furthermore, a retrospective review of adults undergoing treatment for RRP in the United States observed that HPV vaccination was associated with an increase in time between RRP-related surgical procedures and a decrease in the number of procedures required per year (Yiu et al., 2019). Therefore, HPV vaccination has the potential to substantially reduce the burden of RRP.

The impact of HPV vaccination on head and neck cancers is supported by real-world data showing that vaccination is associated with reduced prevalence of oropharyngeal infection with HPV-16, (Mehanna et al., 2018) which accounts for $>80 \%$ of HPV-related oropharyngeal cancers (de Sanjose et al., 2018).

Regarding penile cancer, there is limited clinical data on the impact of HPV vaccination. In an analysis of five randomized double-blind clinical trials of the 9vHPV vaccine in both females and males, no cases $(0 / 1,394)$ of penile intraepithelial neoplasia occurred among males in the 9vHPV arm versus four cases (4/ 1,404) in the control arm (Gardasil, 2018).

The public health impact and cost-effectiveness of GNV with the 9vHPV vaccine has been previously assessed using different transmission dynamic models calibrated to other European settings, including Austria, Italy, Germany, Spain, and France (Mennini et al., 2017; Boiron et al., 2016; Largeron et al., 2017; De La Fuente et al., 2019; Majed et al., 2021). Similar to our findings, these studies reported additional percent reductions in the incidence of cervical cancer over a 100 -year period when GNV with the 9vHPV vaccine was compared to FOV with the 4vHPV vaccine (approximately 17-24\%) (Mennini et al., 2017; Boiron et al., 2016; Largeron et al., 2017; De La Fuente et al., 2019) or FOV with the 9vHPV vaccine (approximately 3 to 7\%) (Mennini et al., 2017; Largeron et al., 2017; De La Fuente et al., 2019; Majed et al., 2021). Additional percent reductions in the incidence of genital warts over a 100-year period ranged from approximately 7 to $29 \%$ for females and from approximately 14 to $44 \%$ for males were observed when GNV with the 9vHPV vaccine was compared to FOV with either the $4 \mathrm{vHPV}$ or 9vHPV vaccine (Mennini et al., 2017; Largeron et al., 2017; De La Fuente et al., 2019; Majed et al., 2021); these reductions were less pronounced than the findings of our analyses, which reported further reductions of approximately $30-65 \%$ for females and approximately 45 to $63 \%$ for males across the three scenarios assessed. Finally, GNV with the 9vHPV vaccine was found to be a cost-effective strategy relative to FOV with the 4vHPV or 9vHPV vaccine, with predicted ICERs $(€ 13,541-€ 30,426)$ falling below or very close to the reference cost-effectiveness thresholds $(€ 30,000$ or $€ 40,000$ ) used for these studies (Mennini et al., 2017; Boiron et al., 2016; Largeron et al., 2017; De La Fuente et al., 2019; Majed et al., 2021). Taken together, these studies along with our findings in the Belgian setting demonstrate the potential for a GNV approach with the 9vHPV vaccine to reduce the burden of $\mathrm{HPV}$-associated disease and be a cost-effective strategy in the European setting.

\section{Limitations}

Several limitations are associated with this modeling approach. The model did not assess possible changes to cervical cancer 
screening methods over the course of the 100 years. The model also did not assess indirect costs, the impact on fertility, as well as neonatal morbidity and mortality due to cervical lesions. In addition, we did not conduct multivariate deterministic sensitivity analyses or probabilistic sensitivity analyses. Currently, disease transmission among populations at high risk, such as MSM and HIV populations, is not included in the model, which may result in underestimating the added benefit of male vaccination compared with an FOV strategy. The presence of groups at high risk can decrease the sensitivity to parameter values, which can lead to relatively small changes in the prevalence of infection despite large changes in transmission rates and infectious periods (Datta et al., 2018). Nevertheless, groups at high risk, such as MSM, often have higher rates of sexually transmitted infections and are thus an important source of infection to the population that must be captured by modeling studies (Datta et al., 2018). In England, selective vaccination of MSM with the 4vHPV vaccine is projected to reduce the incidence of anogenital warts and male HPV-related cancer and is likely to be cost-effective (Lin et al., 2017). Therefore, the inclusion of MSM in future modeling studies will likely increase the benefit of a GNV strategy. Furthermore, the model may overestimate herd immunity, or the protective effects of vaccination at a population level, even among those who were not vaccinated, by assuming coverage in $90 \%$ of women for the Flanders scenario. No migration into Belgium was assumed and the model is not able to account for the historical mixed schedule (i.e., $2 \mathrm{vHPV}$ and $4 \mathrm{vHPV}$ in different regions). In addition, the model incorporated the impact of the vaccine on HPV 31/33/45/52/58 types that cause cervical and anal cancer only and not HPV-related diseases such as vulvar, vaginal, and head and neck cancers. As a result of this exclusion, cases avoided for other cancers and subsequently the value of the 9vHPV vaccine are underestimated. Finally, the amount of underreporting of cancer cases and subsequent deaths due to undiagnosed cancers is not well understood. The model assumes that the death rate for undiagnosed cancer is the same as that of diagnosed cancers. The QALYs gained due to deaths avoided includes deaths due to both diagnosed and undiagnosed cancer and may overestimate the benefit of vaccination.

\section{Conclusion}

Compared with an FOV strategy, a GNV strategy with the 9vHPV vaccine is projected to provide substantial public health (i.e., reductions in HPV-related disease incidence and death) and economic benefits in Belgium when incorporated into the national catch-up program, as well as independent regional vaccination programs in Flanders and Wallonia-Brussels. As a result, a GNV strategy will provide greater benefit in the prevention

\section{REFERENCES}

Agentschap Zorg and Gezondheid (2018). Vaccination against HPV. Available at: https://www.zorg-en-gezondheid.be/vaccinatie-tegen-hpv (Accessed October 28, 2020).

Anic, G. M., Lee, J. H., Villa, L. L., Lazcano-Ponce, E., Gage, C., José C Silva, R., et al. (2012). Risk factors for incident condyloma in a multinational cohort of men: the HIM study. J. Infect. Dis. 205 (5), 789-793. doi:10.1093/infdis/jir851 of HPV-related diseases in the entire male population and additional benefits in females. GNV with the 9vHPV vaccine may also be considered a cost-effective strategy relative to FOV with a broad range of vaccine coverage rates in the regional and national vaccination programs. Future research will look to expand analyses to include populations at high risk, such as MSM, which will truly capture the full impact of a GNV strategy.

\section{DATA AVAILABILITY STATEMENT}

All relevant data is contained within the article. The original contributions presented in the study are included in the article/ Supplementary Material; further inquiries can be directed to the corresponding author.

\section{AUTHOR CONTRIBUTIONS}

SS provided advice and validated the economic evaluation of gender-neutral vaccination with the 9-valent HPV vaccine, and critically reviewed the manuscript. $\mathrm{AB}, \mathrm{BM}$ and $\mathrm{EM}$ conceptualized the manuscript, interpreted the data, and wrote the manuscript. AP adapted and calibrated the model and ran the cost-effectiveness analyses. SJ, SVe and SVa critically reviewed the manuscript.

\section{FUNDING}

This study was sponsored by Merck Sharp and Dohme Corp., a subsidiary of Merck and Co., Inc., Kenilworth, NJ, United States.

\section{ACKNOWLEDGMENTS}

Medical writing assistance was provided by Lei Bai, and Maxwell Chang, of ApotheCom (New York, NY, United States) and was funded by Merck Sharp and Dohme Corp., a subsidiary of Merck and Co., Inc., Kenilworth, NJ, United States.

\section{SUPPLEMENTARY MATERIAL}

The Supplementary Material for this article can be found online at: https://www.frontiersin.org/articles/10.3389/fphar.2021.628434/ full\#supplementary-material.

Annemans, L., Rémy, V., Lamure, E., Spaepen, E., Lamotte, M., Muchada, J. P., et al (2008). Economic burden associated with the management of cervical cancer, cervical dysplasia and genital warts in Belgium. J. Med. Econ. 11 (1), 135-150. doi:10.3111/13696990801961611

Arbyn, M., Haelens, M., Desomer, A., Verdoodt, F., Thiry, N., Francart, G. J., et al. (2015). Cervical cancer screening program and human papillomavirus (HPV) testing, part II: update on HPV primary screening (KCE report 238). Available at: https://kce.fgov.be/sites/default/files/atoms/files/KCE_238_HPV_DNA_ Testing_Report2_.pdf (Accessed October 28, 2020). 
Ault, K. A. (2007). Effect of prophylactic human papillomavirus L1 virus-likeparticle vaccine on risk of cervical intraepithelial neoplasia grade 2 , grade 3 , and adenocarcinoma in situ: a combined analysis of four randomised clinical trials. Lancet 369 (9576), 1861-1868. doi:10.1016/S0140-6736(07)60852-6

Bajos, N., and Boson, M. (2008). Survey on sexuality in France. practices, gender and health. France: Cairn, 612 .

Beachler, D. C., Pinto, L. A., Kemp, T. J., Nyitray, A. G., Hildesheim, A., Viscidi, R., et al. (2018). An examination of HPV16 natural immunity in men who have sex with men (MSM) in the HPV in men (HIM) study. Cancer Epidemiol. Biomarkers Prev. 27 (4), 496-502. doi:10.1158/1055-9965.EPI-17-0853

Belgian Cancer Registry (2017). Cancer fact sheet. head and neck cancer. ICD10: COO-C-14, C30-C32. Available at: https://kankerregister.org/media/docs/ CancerFactSheets/2017/Cancer_Fact_Sheet_HeadandNeckCancer_2017.pdf (Accessed June 1, 2020).

Belgian Cancer Registry (2020). Welcome to the Belgian cancer registry. Available at: http://kankerregister.org/default.aspx?lang=EN (Accessed October 28, 2020).

Belgium National Institute for Health and Disability (INAMI) (2019). National Institute for sickness and disability insurance Brussels, Belgium: Belgium national Institute for health and disability (INAMI). Available at: https://www.inami.fgov.be/fr/Pages/default.aspx. (Accessed March 10, 2021).

Bertram, M. Y., Lauer, J. A., De Joncheere, K., Edejer, T., Hutubessy, R., Kieny, M. P., et al. (2016). Cost-effectiveness thresholds: pros and cons. Bull. World Health Organ. 94 (12), 925-930. doi:10.2471/BLT.15.164418

Boiron, L., Joura, E., Largeron, N., Prager, B., and Uhart, M. (2016). Estimating the cost-effectiveness profile of a universal vaccination programme with a ninevalent HPV vaccine in Austria. BMC Infect. Dis. 16, 153. doi:10.1186/s12879016-1483-5

Brisson, M., Bénard, É., Drolet, M., Bogaards, J. A., Baussano, I., Vänskä, S., et al. (2016). Population-level impact, herd immunity, and elimination after human papillomavirus vaccination: a systematic review and meta-analysis of predictions from transmission-dynamic models. Lancet Public Health 1 (1), e8-e17. doi:10.1016/S2468-2667(16)30001-9

Bruni, L. (2017). ICO/IARC information centre on HPV and cancer (HPV information centre). World Health Organ. Weekly Epidemiol. Rec. 19 (92), 241-268.

Bruni, L., Diaz, M., Barrionuevo-Rosas, L., Herrero, R., Bray, F., Bosch, F. X., et al. (2016). Global estimates of human papillomavirus vaccination coverage by region and income level: a pooled analysis. Lancet Glob. Health 4 (7), e453-63. doi:10.1016/S2214-109X(16)30099-7

Burger, E. A., Sy, S., Nygård, M., Kristiansen, I. S., and Kim, J. J. (2014). Prevention of HPV-related cancers in Norway: cost-effectiveness of expanding the HPV vaccination program to include pre-adolescent boys. PLoS One 9 (3), e89974. doi:10.1371/journal.pone.0089974

Chadha, N. K., Allegro, J., Barton, M., Hawkes, M., Harlock, H., and Campisi, P. (2010). The quality of life and health utility burden of recurrent respiratory papillomatosis in children. Otolaryngol. Head Neck Surg. 143 (5), 685-690. doi:10.1016/j.otohns.2010.08.005

Chesson, H. W., Markowitz, L. E., Hariri, S., Ekwueme, D. U., and Saraiya, M. (2016). The impact and cost-effectiveness of nonavalent HPV vaccination in the United States: estimates from a simplified transmission model. Hum. Vaccin. Immunother. 12 (6), 1363-1372. doi:10.1080/21645515.2016.1140288

Cleemput, I., Kind, P., and Kesteloot, K. (2004). Re-scaling social preference data: implications for modelling. Eur. J. Health Econ. 5 (4), 290-298. doi:10.1007/ s10198-004-0242-5

Cleemput, I., Neyt, M., Thiry, N., De Laet, C., and Leys, M. (2008a). Threshold values for cost-effectiveness in health care (KCE reports 100C). Available at: https://kce.fgov.be/sites/default/files/atoms/files/d20081027396.pdf (Accessed October 28, 2020)

Cleemput, I., Neyt, M., Van de Sande, S., and Thiry, N. (2012). Belgian guidelines for economic evaluations and budget impact analyses: second edition (KCE Report 183C). Available at: https://kce.fgov.be/sites/default/files/atoms/files/ KCE_183_economic_evaluations_second_edition_Report_update.pdf (Accessed October 28, 2020).

Cleemput, I., Van Wilder, P., Vrijens, F., Huybrechts, M., and Ramaekers, D. (2008b). Guidelines for pharmacoeconomic evaluations in Belgium (KCE reports 78A). Available at: https://kce.fgov.be/en/guidelines-forpharmacoeconomic-evaluations-in-belgium (Accessed October 28, 2020).

Conseil Supérieur de la Santé (2017). Vaccination contre les infections causées par le papillomavirus humain Opinion 9181 Conseil Supérieur de la Santé web site: conseil Supérieur de la Santé. Available at: https://www.health.belgium.be/nl/ advies-9181-humaan-papillomavirus (Accessed October 28, 2020). Updated September 25.

Dasbach, E. J., Elbasha, E. H., and Insinga, R. P. (2006). Mathematical models for predicting the epidemiologic and economic impact of vaccination against human papillomavirus infection and disease. Epidemiol. Rev. 28, 88-100. doi:10.1093/epirev/mxj006

D’Hauwers, K. W., Depuydt, C. E., Bogers, J. J., Noel, J. C., Delvenne, P., Marbaix, E., et al. (2012). Human papillomavirus, lichen sclerosus and penile cancer: a study in Belgium. Vaccine 30 (46), 6573-6577. doi:10.1016/j.vaccine.2012. 08.034

Datta, S., Mercer, C. H., and Keeling, M. J. (2018). Capturing sexual contact patterns in modelling the spread of sexually transmitted infections: evidence using Natsal-3. PLoS One 13 (11), e0206501. doi:10.1371/journal.pone.0206501

Datta, S., Pink, J., Medley, G. F., Petrou, S., Staniszewska, S., Underwood, M., et al. (2019). Assessing the cost-effectiveness of HPV vaccination strategies for adolescent girls and boys in the UK. BMC Infect. Dis. 19 (1), 552. doi:10. 1186/s12879-019-4108-y

De La Fuente, J., Hernandez Aguado, J. J., San Martín, M., Ramirez Boix, P., Cedillo Gómez, S., and López, N. (2019). Estimating the epidemiological impact and cost-effectiveness profile of a nonavalent HPV vaccine in Spain. Hum. Vaccin. Immunother. 15, 1949-1961. doi:10.1080/21645515.2018.1560770

de Martel, C., Plummer, M., Vignat, J., and Franceschi, S. (2017). Worldwide burden of cancer attributable to HPV by site, country and HPV type. Int. J. Cancer 141 (4), 664-670. doi:10.1002/ijc.30716

de Sanjosé, S., Serrano, B., Tous, S., Alejo, M., Lloveras, B., Quirós, B., et al. (2018). Burden of human papillomavirus (HPV)-related cancers attributable to HPVs 6/11/16/18/31/33/45/52 and 58. JNCI Cancer Spectr. 2 (4), pky045. doi:10.1093/ jncics/pky045

Dominiak-Felden, G., Cohet, C., Atrux-Tallau, S., Gilet, H., Tristram, A., and Fiander, A. (2013). Impact of human papillomavirus-related genital diseases on quality of life and psychosocial wellbeing: results of an observational, healthrelated quality of life study in the UK. BMC Public Health 13, 1065. doi:10.1186/ 1471-2458-13-1065

Dominiak-Felden, G., Gobbo, C., and Simondon, F. (2015). Evaluating the early benefit of quadrivalent HPV vaccine on genital warts in Belgium: a cohort study. PLoS One 10 (7), e0132404. doi:10.1371/journal.pone.0132404

Donne, A. J., Hampson, L., Homer, J. J., and Hampson, I. N. (2010). The role of HPV type in recurrent respiratory papillomatosis. Int. J. Pediatr. Otorhinolaryngol. 74 (1), 7-14. doi:10.1016/j.ijporl.2009.09.004

Donovan, B., and Callander, D. (2018). Juvenile-onset recurrent respiratory papillomatosis: the benefits of quadrivalent human papillomavirus vaccination continue to accrue. J. Infect. Dis. 217 (2), 177-178. doi:10.1093/ infdis/jix499

Dunne, E. F., Unger, E. R., Sternberg, M., McQuillan, G., Swan, D. C., Patel, S. S., et al. (2007). Prevalence of HPV infection among females in the United States. J. Am. Med. Assoc. 297 (8), 813-819. doi:10.1001/jama.297.8.813

Elbasha, E. H., and Dasbach, E. J. (2010). Impact of vaccinating boys and men against HPV in the United States. Vaccine 28 (42), 6858-6867. doi:10.1016/j. vaccine. 2010.08.030

Elbasha, E. H., Dasbach, E. J., and Insinga, R. P. (2007). Model for assessing human papillomavirus vaccination strategies. Emerg. Infect. Dis. 13 (1), 28-41. doi:10. 3201/eid1301.060438

Favaretti, C., Kheiraoui, F., de Waure, C., Poscia, A., Sacchini, D., De Vincenzo, R., et al. (2017). Il vaccinoanti-HPV 9-valente: report di Health technology assessment (HTA). Quaderni Ital. J. Public Health 6 (5), 142.

Forman, D., de Martel, C., Lacey, C. J., Soerjomataram, I., Lortet-Tieulent, J., Bruni, L., et al. (2012). Global burden of human papillomavirus and related diseases. Vaccine 30 (Suppl. 5), F12-F23. doi:10.1016/j.vaccine.2012.07.055

Fortes, H. R., von Ranke, F. M., Escuissato, D. L., Araujo Neto, C. A., Zanetti, G., Hochhegger, B., et al. (2017). Recurrent respiratory papillomatosis: a stateof-the-art review. Respir. Med. 126, 116-121. doi:10.1016/j.rmed.2017. 03.030 
Frisch, M., Smith, E., Grulich, A., and Johansen, C. (2003). Cancer in a populationbased cohort of men and women in registered homosexual partnerships. Am. J. Epidemiol. 157 (11), 966-972. doi:10.1093/aje/kwg067

Gardasil (2018). Gardasil [prescribing information] 10/2018. Whitehouse Station, NJ: Merck Sharp \& Dohme Corp.

Garland, S. M., Hernandez-Avila, M., Wheeler, C. M., Perez, G., Harper, D. M., Leodolter, S., et al. (2007). Quadrivalent vaccine against human papillomavirus to prevent anogenital diseases. N. Engl. J. Med. 356 (19), 1928-1943. doi:10. 1056/NEJMoa061760

Garland, S. M., Steben, M., Sings, H. L., James, M., Lu, S., Railkar, R., et al. (2009). Natural history of genital warts: analysis of the placebo arm of 2 randomized phase III trials of a quadrivalent human papillomavirus (types 6, 11, 16, and 18) vaccine. J. Infect. Dis. 199 (6), 805-814. doi:10.1086/597071

Geert, T. (2018). The HPV vaccination programme in Flanders: Flanders, State of the art. Available at: http://www.vaccineconfidence.org/wp-content/uploads/ 2017/06/9_-Geert-top.pdf (Accessed June 1, 2020).

Giuliano, A. R., Lazcano-Ponce, E., Villa, L. L., Flores, R., Salmeron, J., Lee, J. H., et al. (2008). The human papillomavirus infection in men study: human papillomavirus prevalence and type distribution among men residing in Brazil, Mexico, and the United States. Cancer Epidemiol. Biomarkers Prev. 17 (8), 2036-2043. doi:10.1158/1055-9965.EPI-08-0151

Giuliano, A. R., Nyitray, A. G., Kreimer, A. R., Pierce Campbell, C. M., Goodman, M. T., Sudenga, S. L., et al. (2015). EUROGIN 2014 roadmap: differences in human papillomavirus infection natural history, transmission and human papillomavirus-related cancer incidence by gender and anatomic site of infection. Int. J. Cancer 136 (12), 2752-2760. doi:10.1002/ijc.29082

Giuliano, A. R., Palefsky, J. M., Goldstone, S., Moreira, E. D., Jr., Penny, M. E., Aranda, C., et al. (2011). Efficacy of quadrivalent HPV vaccine against HPV infection and disease in males. N. Engl. J. Med. 364 (5), 401-411. doi:10.1056/ NEJMoa0909537

Global Burden of Disease Cancer Collaboration; Fitzmaurice, C., Dicker, D., Pain, A., Hamavid, H., Moradi-Lakeh, M., MacIntyre, MF, et al. (2015). The global burden of cancer 2013. JAMA Oncol. 1 (4), 505-527. doi:10.1001/jamaoncol. 2015.0735

Grisar, K., Dok, R., Schoenaers, J., Dormaar, T., Hauben, E., Jorissen, M., et al. (2016). Differences in human papillomavirus-positive and -negative head and neck cancers in Belgium: an 8-year retrospective, comparative study. Oral Surg. Oral Med. Oral Pathol. Oral Radiol. 121 (5), 456-460. doi:10.1016/j.oooo.2015. 10.035

Hartwig, S., St Guily, J. L., Dominiak-Felden, G., Alemany, L., and de Sanjosé, S. (2017). Estimation of the overall burden of cancers, precancerous lesions, and genital warts attributable to 9-valent HPV vaccine types in women and men in Europe. Infect. Agents Cancer 12, 19. doi:10.1186/s13027-017-0129-6

Hartwig, S., Syrjänen, S., Dominiak-Felden, G., Brotons, M., and Castellsagué, X. (2012). Estimation of the epidemiological burden of human papillomavirusrelated cancers and non-malignant diseases in men in Europe: a review. BMC Cancer 12, 30. doi:10.1186/1471-2407-12-30

Health Information and Quality Authority (2018). Guidelines for the economic evaluation of health technologies in Ireland. Updated September 28, 2020. Available at: https://www.hiqa.ie/reports-and-publications/health-technologyassessment/guidelines-economic-evaluation-health (Accessed June 1, 2020). Published January 2018.

Herrero, R., Quint, W., Hildesheim, A., Gonzalez, P., Struijk, L., Katki, H. A., et al. (2013). Reduced prevalence of oral human papillomavirus (HPV) 4 years after bivalent HPV vaccination in a randomized clinical trial in Costa Rica. PLoS One 8 (7), e68329. doi:10.1371/journal.pone.0068329

Hu, D., and Goldie, S. (2008). The economic burden of noncervical human papillomavirus disease in the United States. Am. J. Obstet. Gynecol. 198 (5), 500.e1-7. doi:10.1016/j.ajog.2008.03.064

Hughes, O., Tsikoudas, A., Auerbach, R., Barr, A., Bateman, N., and Blane, Y. S. (2011). The burden of recurrent respiratory papillomatosis in the United Kingdom: results from the BAPO and ENT-UK national survey. Clin. Otolaryngol. 36 (4), 409. doi:10.1111/j.1749-4486.2011.02363.x

Institut national d'Etudes demographiques (2009). Flemings and walloons Paris, France: Institut national d'études démographiques; 2009. updated August 2019. Available at: https://www.ined.fr/en/everything_about_population/demographicfacts-sheets/focus-on/flemish-walloon/ (Accessed October 28, 2020).
Joint Committee on Vaccination and Immunisation (2018). Statement on HPV vaccination. Available at: https://assets.publishing.service.gov.uk/government/ uploads/system/uploads/attachment_data/file/726319/JCVI_Statement_on_ HPV_vaccination_2018.pdf (Accessed October 28, 2020).

Joura, E. A., Giuliano, A. R., Iversen, O. E., Bouchard, C., Mao, C., Mehlsen, J., et al. (2015). A 9-valent HPV vaccine against infection and intraepithelial neoplasia in women. N. Engl. J. Med. 372 (8), 711-723. doi:10.1056/ NEJMoa1405044

Joura, E. A., Leodolter, S., Hernandez-Avila, M., Wheeler, C. M., Perez, G., Koutsky, L. A., et al. (2007). Efficacy of a quadrivalent prophylactic human papillomavirus (types $6,11,16$, and 18) $\mathrm{L} 1$ virus-like-particle vaccine against high-grade vulval and vaginal lesions: a combined analysis of three randomised clinical trials. Lancet 369 (9574), 1693-1702. doi:10.1016/S0140-6736(07) 60777-6

KCE (2019). Vaccinate boys against the papillomavirus? [press release]. January 31, 2019. Available at: https://kce.fgov.be/fr/vacciner-les-gar\%C3\%A7ons-contrele-papillomavirus (Accessed October 28, 2020).

Kind, A. B., Pavelyev, A., Kothari, S., El Mouaddin, N., Schmidt, A., Morais, E., et al. (2020). Assessing the epidemiological impact on cervical cancer of switching from 4-valent to 9-valent HPV vaccine within a gender-neutral vaccination programme in Switzerland. BMC Public Health 20 (1), 671. doi:10.1186/s12889020-08840-0

Kmietowicz, Z. (2018). Boys in England to get HPV vaccine from next year. $B r$. Med. J. 362, k3237. doi:10.1136/bmj.k3237

La Belgique en Chiffres (2018). New slight progress in the life expectancy of the Belgian population. Available at: https://statbel.fgov.be/fr/themes/population/ mortalite-et-esperance-de-vie/tables-de-mortalite-et-esperance-de-vie\#news (Accessed October 28, 2020).

Largeron, N., Petry, K. U., Jacob, J., Bianic, F., Anger, D., and Uhart, M. (2017). An estimate of the public health impact and cost-effectiveness of universal vaccination with a 9-valent HPV vaccine in Germany. Expert Rev. Pharmacoecon. Outcomes Res. 17 (1), 85-98. doi:10.1080/14737167.2016. 1208087

Lin, A., Ong, K. J., Hobbelen, P., King, E., Mesher, D., Edmunds, W. J., et al. (2017). Impact and cost-effectiveness of selective human papillomavirus vaccination of men who have sex with men. Clin. Infect. Dis. 64 (5), 580-588. doi:10.1093/cid/ ciw845

Majed, L., Bresse, X., El Mouaddin, N., Schmidt, A., Daniels, V. J., Pavelyev, A., et al. (2021). Public health impact and cost-effectiveness of a nine-valent gender-neutral HPV vaccination program in France. Vaccine 39 (2), 438-446. doi:10.1016/j.vaccine.2020.10.089

Matsuzaki, H., Makiyama, K., Hirai, R., Suzuki, H., Asai, R., and Oshima, T. (2020). Multi-year effect of human papillomavirus vaccination on recurrent respiratory papillomatosis. Laryngoscope 130 (2), 442-447. doi:10.1002/lary.27993

Mauz, P. S., Schäfer, F. A., Iftner, T., and Gonser, P. (2018). HPV vaccination as preventive approach for recurrent respiratory papillomatosis-a 22-year retrospective clinical analysis. BMC Infect. Dis. 18 (1), 343. doi:10.1186/ s12879-018-3260-0

Mehanna, H., Bryant, T. S., Babrah, J., Louie, K., Bryant, J. L., Spruce, R. J., et al. (2019). Human papillomavirus (HPV) vaccine effectiveness and potential herd immunity for reducing oncogenic oropharyngeal HPV-16 prevalence in the United Kingdom: a cross-sectional study. Clin. Infect. Dis. 69 (8), 1296-1302. doi:10.1093/cid/ciy1081

Mennini, F. S., Bonanni, P., Bianic, F., de Waure, C., Baio, G., Plazzotta, G., et al. (2017). Cost-effectiveness analysis of the nine-valent HPV vaccine in Italy. Cost Eff. Resour. Alloc. 15, 11. doi:10.1186/s12962-017-0073-8

Murillo, R., Almonte, M., Pereira, A., Ferrer, E., Gamboa, O. A., Jerónimo, J., et al. (2008). Cervical cancer screening programs in Latin America and the Caribbean. Vaccine 26 (Suppl. 11), L37-L48. doi:10.1016/j.vaccine.2008.06.013

National Institute for Health and Care Excellence (2014). Process and methods guides-developing NICE guidelines: the manual. Available at: https://www. nice.org.uk/media/default/about/what-we-do/our-programmes/developingnice-guidelines-the-manual.pdf (Accessed October 28, 2020).

Ndiaye, C., Mena, M., Alemany, L., Arbyn, M., Castellsagué, X., Laporte, L., et al. (2014). HPV DNA, E6/E7 mRNA, and p16INK4a detection in head and neck cancers: a systematic review and meta-analysis. Lancet Oncol. 15 (12), 1319-1331. doi:10.1016/S1470-2045(14)70471-1 
Ng, S. S., Hutubessy, R., and Chaiyakunapruk, N. (2018). Systematic review of costeffectiveness studies of human papillomavirus (HPV) vaccination: 9-Valent vaccine, gender-neutral and multiple age cohort vaccination. Vaccine 36 (19), 2529-2544. doi:10.1016/j.vaccine.2018.03.024

Novakovic, D., Cheng, A. T. L., Zurynski, Y., Booy, R., Walker, P. J., Berkowitz, R., et al. (2018). A prospective study of the incidence of juvenile-onset recurrent respiratory papillomatosis after implementation of a national HPV vaccination program. J. Infect. Dis. 217 (15), 208-212. doi:10.1093/ infdis/jix498

Nyitray, A. G., Carvalho da Silva, R. J., Baggio, M. L., Lu, B., Smith, D., Abrahamsen, M., et al. (2011). Age-specific prevalence of and risk factors for anal human papillomavirus (HPV) among men who have sex with women and men who have sex with men: the HPV in men (HIM) study. J. Infect. Dis. 203 (1), 49-57. doi:10.1093/infdis/jiq021

Omland, T., Akre, H., Vårdal, M., and Brøndbo, K. (2012). Epidemiological aspects of recurrent respiratory papillomatosis: a population-based study. Laryngoscope 122 (7), 1595-1599. doi:10.1002/lary.23327

Palefsky, J. M., Giuliano, A. R., Goldstone, S., Moreira, E. D., Jr., Aranda, C., Jessen, H., et al. (2011). HPV vaccine against anal HPV infection and anal intraepithelial neoplasia. N. Engl. J. Med. 365 (17), 1576-1585. doi:10.1056/ NEJMoa1010971

Qendri, V., Bogaards, J. A., Baussano, I., Lazzarato, F., Vänskä, S., and Berkhof, J. (2020). The cost-effectiveness profile of sex-neutral HPV immunisation in European tender-based settings: a model-based assessment. Lancet Public Health 5 (11), e592-e603. doi:10.1016/S2468-2667(20)30209-7

Schiffman, M., and Castle, P. E. (2003). Human papillomavirus: epidemiology and public health. Arch. Pathol. Lab. Med. 127 (8), 930-934. doi:10.1043/15432165(2003)127

STATBEL (2019). Population by residence, nationality (Belgian/non-Belgian), marital status, age and gender. Available at: https://bestat.statbel.fgov.be/ bestat $/$ crosstable.xhtml? datasource $=65 e e 413 \mathrm{~b}-3859-4 \mathrm{c} 6 \mathrm{f}-\mathrm{a} 847-09 \mathrm{~b} 631766 \mathrm{fa} 7$ (Accessed June 1, 2020).

Sullivan, P. W., Slejko, J. F., Sculpher, M. J., and Ghushchyan, V. (2011). Catalogue of EQ-5D scores for the United Kingdom. Med. Decis. Making 31 (6), 800-804. doi:10.1177/0272989X11401031

Szende, A., Janssen, B., and Cabases, J. (2014). Self-reported population health: an international perspective based on EQ-5D. Dordrecht, Heidelberg, New York, NY, London, United Kingdom: Springer, 210.

Szende, A., and Williams, A. (2004). Measuring self-reported population health: an International perspective based on EQ-5D. Dordrecht, Heidelberg, New York, NY, London, United Kingdom: EuroQol Research Foundation.

Takla, A., Wiese-Posselt, M., Harder, T., Meerpohl, J. J., Röbl-Mathieu, M., Terhardt, M., et al. (2018). Background paper for the recommendation of HPV vaccination for boys in Germany. Bundesgesundheitsblatt Gesundheitsforschung Gesundheitsschutz 61 (9), 1170-1186. doi:10.1007/ s00103-018-2791-2

The Belgian Health Care Knowledge Centre (KCE) (2007). HPV vaccination for the prevention of cervical cancer in Belgium: health technology assessment. KCE reports vol 64C. Available at: https://kce.fgov.be/sites/ default/files/atoms/files/d20071027343.pdf (Accessed October 28, 2020).
Thiry, N., Gerkens, S., Cornelis, J., Jespers, V., and Hanquet, G. (2019). Costeffectiveness analysis of HPV vaccination of boys in Belgium (KCE Reports 308). Available at: https://kce.fgov.be/en/cost-effectiveness-analysis-of-hpvvaccination-of-boys-in-belgium (Accessed October 28, 2020).

Tjalma, W., Brasseur, C., Top, G., Ribesse, N., Morales, I., and Van Damme, P. A. (2018). HPV vaccination coverage in the federal state of Belgium according to regions and their impact. Facts Views Vis. Obgyn 10 (2), 101-105.

Tsakeu, E., Petit, C., and Chevalier, P. (2016). Hospitalization cost and length of stay associated with HPV-related diseases. Value Health 19 (7), A611. doi:10. 1016/j.jval.2016.09.1525 Abstract PHS45.

Vaccination Info Belgium (2020a). Boys also vaccinated against HPV. Updated March 2, 2020. Available at: https://www.vaccination-info.be/les-garconsaussi-vaccines-contre-le-hpv/ (Accessed October 28, 2020).

Vaccination Info Belgium (2020b). Vaccination against human papillomavirus. updated March 2, 2020. Available at: https://www.vaccination-info.be/maladie/ infections-a-papillomavirus-humains-hpv/ (Accessed October 28, 2020).

Wilkin, T. J., Chen, H., Cespedes, M. S., Leon-Cruz, J. T., Godfrey, C., Chiao, E. Y., et al. (2018). A randomized, placebo-controlled trial of the quadrivalent human papillomavirus vaccine in human immunodeficiency virus-infected adults aged 27 years or older: AIDS clinical trials group protocol A5298. Clin. Infect. Dis. 67 (9), 1339-1346. doi:10.1093/cid/ciy274

World Health Organization (2020). Global strategy to accelerate the elimination of cervical cancer as a public health problem. Geneva, Switzerland: World health Organization. Available at: https://apps.who.int/iris/rest/bitstreams/1315304/ retrieve (Accessed February 2, 2021).

Yiu, Y., Fayson, S., Smith, H., and Matrka, L. (2019). Implementation of routine HPV vaccination in the management of recurrent respiratory papillomatosis. Ann. Otol Rhinol Laryngol. 128 (4), 309-315. doi:10. $1177 / 0003489418821695$

Conflict of Interest: SS has been involved in research and in advisory boards related to the economic evaluation of vaccines funded by MSD, GSK and Pfizer. BM, SJ, and EM are employees of MSD. SVe was an employee of MSD at the time of development of this manuscript and is currently an employee of UCB. AP and SVa are employees of Merck and Co., Inc. BM and SVa also hold stock in Merck and Co., Inc.

The remaining author declares that the research was conducted in the absence of any commercial or financial relationships that could be construed as a potential conflict of interest.

The handling editor declared a past co-authorship with one of the authors (SS).

Copyright (C) 2021 Simoens, Bento-Abreu, Merckx, Joubert, Vermeersch, Pavelyev, Varga and Morais. This is an open-access article distributed under the terms of the Creative Commons Attribution License (CC BY). The use, distribution or reproduction in other forums is permitted, provided the original author(s) and the copyright owner(s) are credited and that the original publication in this journal is cited, in accordance with accepted academic practice. No use, distribution or reproduction is permitted which does not comply with these terms. 